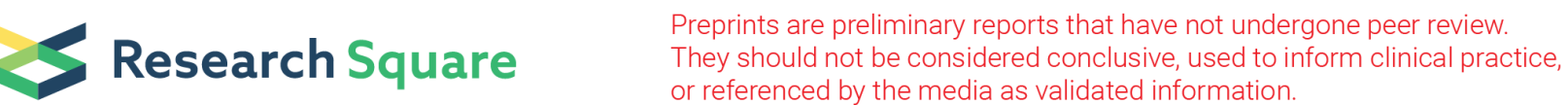

\section{HO-1/Bmmscs Perfusion Using a Normothermic Machine Perfusion System Reduces the Acute Rejection of DCD Liver Transplantation by Regulating NKT Cell Co-Inhibitory Receptors}

\section{Huan Cao}

Tianjin First Central Hospital Clinic Institute, Tianjin Medical University.

\section{Longlong Wu}

School of Medicine, Nankai University.

\section{Xuan Tian}

School of Medicine, Nankai University.

\section{Weiping Zheng}

Tianjin First Central Hospital and NHC Key Laboratory of Critical Care Medicine,Tianjin.

\section{Mengshu Yuan}

Tianjin First Central Hospital Clinic Institute, Tianjin Medical University.

\section{Xiang Li}

Tianjin First central Hospital Clinic Institute, Tianjin Medical University.

\section{Xiaorong Tian}

Tianjin First Central Hospital Clinic Institute, Tianjin Medical University.

\section{Yuxin Wang}

Tianjin First Central Hospital Clinic Institute, Tianjin Medical University.

\section{Hongli Song ( $\nabla$ hlsong26@163.com )}

Tianjin First Central Hospital and Tianjin Key Laboratory of Organ Transplantation

\section{Zhongyang Shen}

Tianjin First Central Hospital and Key Laboratory of Transplant Medicine, Chinese Academy of Medical Sciences, Tianjin.

\section{Research}

Keywords: acute rejection, bone marrow mesenchymal stem cells, natural killer T cells, liver transplantation, normothermic machine perfusion

Posted Date: April 14th, 2021

DOI: https://doi.org/10.21203/rs.3.rs-380793/v1 
License: (c) (i) This work is licensed under a Creative Commons Attribution 4.0 International License. Read Full License

Version of Record: A version of this preprint was published at Stem Cell Research \& Therapy on November 24th, 2021. See the published version at https://doi.org/10.1186/s13287-021-02647-5. 


\section{Abstract}

Background: Liver transplantation (LT) represents the most effective treatment for many end-stage liver diseases. While donation after cardiac death (DCD) donor livers are used due to organ shortage, acute rejection (ACR) remains an important risk factor affecting the survival of recipients following transplantation. Although immunosuppressive agents can be used, they are associated with complications. Bone marrow mesenchymal stem cells (BMMSCs) are used in the treatment of organ transplantation; however, there is limited colonization in the target organs and a short survival time following BMMSCs application. Thus, an optimized BMMSCs application method is required to suppress immune rejection and promote the long-term survival of allogeneic liver transplant recipients.

Methods: BMMSCs were isolated and modified with heme oxygenase 1 (HO-1) gene. HO-1/BMMSCs were perfused into the donor liver in vitro using a normothermic machine perfusion (NMP) system, followed by LT. The severity of ACR was evaluated based on the liver histopathology. Gene chip technology was used to detect differential gene expression, and the flow cytometry was used to analyze changes in natural killer (NK) T cells.

Results: NMP can induce BMMSCs to colonize the donor liver during in vitro preservation, and the survival of HO-1/BMMSCs in the liver grafts was significantly longer than that of BMMSCs. When the donor liver contained HO-1/BMMSCs, the ACR is obviously controlled, and the survival time was significantly prolonged. The application of HO-1/BMMSCs reduces the number of NKT cells in the liver grafts, increases the expression of the NKT cell co-inhibitory receptors, and reduces the level of NKT cell expression of IFN- $\gamma$. Thus, NK cell and $\mathrm{CD}^{+}{ }^{+} \mathrm{T}$ cell activation was inhibited, which reduced acute of rejection of the transplanted liver.

Conclusions: The NMP system preserves the DCD donor liver in vitro, and also allows large quantities of BMMSCs to colonize the liver. HO-1-modified BMMSCs are able to improve and prolong the local immunosuppressive effect of BMMSCs following transplantation by reducing the number of NKT cells and up-regulating NKT cell co-inhibitory receptor expression. This results in the transmission of inhibitory signals to NKT cells, reduced NKT cell IFN-y levels, and the inhibition of ACR.

\section{Background}

Liver transplantation (LT) is the most effective means of treating end-stage liver diseases (e.g., cirrhosis and liver cancer) [1]; however, the development of LT also faces some challenges. First, with the maturation of LT techniques, the number of LT procedures has increased annually. In conjunction with a dramatic increase in the number of patients waiting for transplantation, there is a significant shortage of donors. In an attempt to alleviate this organ shortage, marginal donors have inevitably been used for transplantation [2, 3]. Donation after cardiac death (DCD) donor livers comprise a substantial source of marginal donor livers, and the rational use of DCD donor livers can significantly expand the donor pool [4, 5]. However, static cold storage (SCS) methods are ineffective at preserving DCD donor livers, which can 
lead to an increased probability of serious postoperative complications and will affect the prognosis of the transplantation $[4,6]$.

Although it has been recognized that the liver is an immunologically privileged organ in solid organ transplantation, the probability of acute rejection (ACR) without the application of an effective immunosuppressive regimen following transplantation remains as high as $20 \%-40 \%$, which can seriously affect recipient survival [7]. Immunosuppressive regimens, represented by tacrolimus as the main calcineurin inhibitor, can significantly attenuate rejection after transplantation and improve the prognostic outcome of LT [8]. Despite the application of immunosuppressive agents, cases of ACR in some patients remain the main cause of graft dysfunction and an important risk factor for recipient survival following transplantation [9]. To prevent the occurrence of complications (e.g., graft loss) due to the development of acute and chronic rejection, long-term immunosuppressant use is required. In the case of long-term immunosuppressive regimens, the incidence of their associated complications (e.g., renal injury, abnormal metabolic syndrome, and de novo tumors) increases, and their long-term outcomes remain unsatisfactory $[10,11]$. To address the drawbacks of ACR and complications associated with lifelong immunosuppressive applications, there is a need to explore an effective immunomodulatory means to induce immune tolerance in $L T$, mitigate ACR, and reduce or completely avoid the use of immunosuppressive agents.

Bone marrow mesenchymal stem cells (BMMSCs) are suitable for the treatment of various congenital and acquired diseases due to their multilineage differentiation potential, paracrine effects favoring tissue reconstruction and immune regulation, as well as high accessibility and expansion capacity $[12,13]$. However, substantial evidence suggests that following a systemic injection, most BMMSCs reside in the capillary beds of various tissues, particularly the lungs, which both compromises therapeutic efficacy and also poses a risk of pulmonary embolism [14]. Therefore, topical application is the preferred method of BMMSCs administration and BMMSCs therapy also has the disadvantage of poor survival in target organs [15]. We previously used a heme oxygenase-1 (HO-1) gene transfection of BMMSCs to improve the viability of BMMSCs in target organs and to enhance and optimize the effect of BMMSCs $[16,17]$. In addition, normothermic machine perfusion (NMP) is a novel method of organ preservation that maintains a temperature close to the physiological state and supplies oxygen and energy substrates in vitro. This provides improved protection of DCD liver grafts and the prognosis of DCD LT. Our previous studies found that NMP-perfused HO-1/BMMSCs heavily colonize the transplanted liver and exhibit excellent protection $[17,18]$.

Previous studies have confirmed the involvement of both natural and acquired immunity in liver transplant rejection [19]; however, the mechanisms that induce immune tolerance or suppress rejection remain unclear. Natural killer (NK) T cells have the characteristics of both innate and acquired immune cells. Moreover, NKT cells are abundant in the liver sinusoids, and studies have shown that they significantly increase following LT [20]. NKT cells express a T cell receptor (TCR), NK cell receptors, and respond to self or non-self lipid antigens loaded on CD1d molecules [21, 22]. Once activated, NKT cells can promote the rapid production and release of a large number of inflammatory cytokines within a few 
hours, which regulate the function of many immune cells [23]. For example, interleukin (IL)-2, interferon (IFN)- $y$, and tumor necrosis factor (TNF)-a induce pro-inflammatory Th (T helper, Th) 1 cell responses [24], whereas IL-4, IL-5, IL-6, IL-10 and IL-13 IL-6, IL-10, and IL-13 promote pro-inflammatory Th2 cell responses [25]. There is increasing evidence suggesting that NKT cells are essential in the regulation of autoimmune responses and IFN- $\gamma$ production activates NK cell and $\mathrm{CD} 8^{+} \mathrm{T}$ cell immune responses, which are closely associated with the development of ACR of LT [26]; however, the effects of NKT cells on ACR of LT remain to be elucidated.

In this study, we used NMP to infuse HO-1/BMMSCs into the donor liver through the portal vein during its in vitro preservation under the premise of protecting the DCD donor liver in vitro. HO-1/BMMSCs were colonized in the donor liver and the effect of HO-1/BMMSCs on NKT cells and their inhibitory receptors was observed in the DCD liver grafts in rejection and the associated mechanism was explored. The findings of this study will provide a novel method by which optimized BMMSCs regulate the immunosuppressive state of transplantation and prolong the survival time of the recipient.

\section{Methods}

\section{Animals}

The experimental animals were provided by Beijing Vital River Laboratory Animal Technology Co. Ltd. (Beijing, China). Male Lewis rats ( $7-8$ weeks old, $200 \mathrm{~g}-220 \mathrm{~g}$ ) were used as donors, and male Brown Norway rats ( $8-9$ weeks old, $220 \mathrm{~g}-240 \mathrm{~g})$ were used as the recipients. The rats were divided into the following six groups according to the different liver treatments: sham operated (Sham) group; SCS group; NMP group; NMP + BMMSCs (BMP) group; NMP + HO-1/BMMSCs (HMP) group; and NMP + FK506 (FK506) group. A total of 36 (6 rats/group) recipients were used for the survival analysis, and the other 72 recipients were used for postoperative $(7 d, n=6 ; 14 d, n=6)$ specimen collection. The animals were housed in a standard laboratory animal room at a constant temperature $\left(22^{\circ} \mathrm{C} \pm 1^{\circ} \mathrm{C}\right), 60 \%$ relative humidity, $12 \mathrm{~h} / 12 \mathrm{~h}$ light-dark cycle, and free access to water and food. All animal protocols were based on the National Institutes of Health "Guide for the Care and Use of Laboratory Animals" (National Institutes of Health publication $85-23$, Bethesda, MD). Efforts were made to minimize the number of animals used and any discomfort encountered, and all procedures were approved by the Ethics Committee of Tianjin First Central Hospital (license number: 2016-03-A1).

\section{Preparation and characterization of H0-1/BMMSCs}

BMMSCs were prepared in accordance with our previous methods $[16,17]$. Briefly, the femurs and tibias were removed under aseptic conditions after the rats had been executed and sterilized, and BMMSCs were extracted using a whole bone marrow apposition screening method, and perfused or transfected with HO-1 after culturing to the third generation. HO-1/BMMSCs were prepared by transfecting HO-1 using an adenovirus (Genechem, Shanghai, China) when the cells were in a stable state. The differentiation ability of the HO-1/BMMSCs was determined via in vitro osteogenic and lipogenic differentiation with antibodies against CD29, CD34, CD45, CD90, RT1A, and RT1B (Biolegend, CA, US) staining was used to 
identify the molecular phenotype using flow cytometry. qRT-PCR and Western blot were used to determine whether HO-1 expression was elevated.

\section{DCD model}

The rats were anesthetized and placed on a warming pad, the abdomen was opened along the median abdomen, the liver, inferior vena cava and portal vein were freed, and heparin (Solarbio, Beijing, China) (1 $\mathrm{U} / \mathrm{g}$ body weight) was injected from the dorsal penile vein. After $10 \mathrm{~min}$, the diaphragm was cut and the heart was compressed to promote cardiac arrest, simulating the process of circulatory death in vivo, and the abdominal temperature was maintained with warm saline at $37^{\circ} \mathrm{C}\left(\right.$ range: $35^{\circ} \mathrm{C}-37^{\circ} \mathrm{C}$ ) for $30 \mathrm{~min}$.

\section{H0-1/BMMSCs combined with NMP for in vitro preservation of donor livers}

The NMP system primarily consists of an organ compartment, peristaltic pump, filter, membrane oxygenator and oxygen supply system, as well as a temperature and pressure sensor (Figure S1A and B). The donor liver was perfused with portal vein, and the perfusion temperature was maintained at $36^{\circ} \mathrm{C}-$ $38^{\circ} \mathrm{C}$. The main components of the perfusion fluid consist of $20 \%$ fetal bovine serum (FBS, Biowest, Nuaillé, France) containing, $60 \mathrm{~mL}$ Dulbecco's modified Eagle's medium (DMEM)/F12 (Gibco, Thermo Scientific, Waltham, MA, USA), $20 \mathrm{~mL}$ rat blood, $100 \mathrm{U} / \mathrm{mL}$ penicillin (Gibco), $100 \mu \mathrm{g} / \mathrm{mL}$ streptomycin (Gibco), and $5 \mathrm{U} / \mathrm{mL}$ heparin (Gibco) [17]. All of the donor livers were flushed with $10 \mathrm{~mL}$ University of Wisconsin (UW) solution through the portal vein before performing machine perfusion, and the SCS group was subjected to static cold storage with UW solution. NMP livers were simply mechanically perfused, the BMP group was perfused with $1 \times 10^{7}$ BMMSCs through the portal vein, the HMP group was perfused with $1 \times 10^{7} \mathrm{HO}-1 / \mathrm{BMMSC}$ through the portal vein (all BMMSCs were perfused into the liver through a $100-\mu \mathrm{m}$ pore size filter). The livers in the FK506 group were mechanically perfused and $0.1 \mathrm{mg} / \mathrm{kg} \mathrm{FK506} \mathrm{was}$ administered intragastrically daily following surgery [27]. The donor livers were stored in vitro for $4 \mathrm{~h}$ before transplantation.

\section{Orthotopic LT}

The rats were subjected to the orthotopic LT technique based on Kamada's "two-cuff method" [17, 28]. The operator was a surgical professional and underwent substantial training in the early stages. The anhepatic phase is controlled at $19 \pm 1 \mathrm{~min}$. After transplantation, the rats received $2 \mathrm{~mL}$ Ringer's lactate and were rewarmed in a postoperative incubator for $30 \mathrm{~min}$. On days 7 and 10 after LT, the recipient was euthanized by an intraperitoneal injection with an overdose of pentobarbital $(150 \mathrm{mg} / \mathrm{kg})$, and the spleen, blood, and liver grafts were obtained.

\section{BMMSCs tracing}

To demonstrate that BMMSCs can colonize in the liver grafts, an adenovirus expressing green fluorescent protein (GFP) (Genechem) was used to transfect the BMMSCs using NMP perfusion into the liver. The level of fluorescence expression in the liver was observed using an in vivo imaging system (PerkinElmer, 
CA, USA) after LT. After the recipient was sacrificed, the liver grafts were obtained, and frozen sections were prepared to observe BMMSCs colonization in the liver tissue under a fluorescence microscope.

\section{Histopathology}

The livers were fixed in a $10 \%$ neutral formalin solution and sections of each of the samples were stained with hematoxylin-eosin (HE) to visualize transplanted liver histopathology or for terminal deoxynucleotidyl transferase dUTP nick end labeling (TUNEL) in accordance with the methods provided by the manufacturer of a commercial kit. The rejection activity index (RAl) was used to evaluate the degree of ACR.

\section{Liver function test}

The levels of serum alanine aminotransferase (ALT), aspartate aminotransferase (AST), alkaline phosphatase (ALP), glutamyl transpeptidase (GGT), total bilirubin (TBil), and serum albumin (ALB) were detected with an automatic biochemical analyzer (Cobas 800, Roche, Basel, Switzerland).

\section{Enzyme-linked immunosorbent assay (ELISA)}

The serum and cell culture supernatant were analyzed for the concentration of cytokines, and the serum content of IL-2, IFN- $\gamma$, and TNF-a were detected in the serum in accordance with the manufacturer's instructions of the commercial ELISA kit (MultiSciences Biotech Co., Hangzhou, China).

\section{Western blot}

The manufacturer's instructions (Solarbio, Beijing, China) of a commercial kit were used to extract the total cell protein using cell lysates, after which a Western blot was performed. The detailed experimental methods were performed as described in our previously published literature [16]. Using $\beta$-actin as a control, the membranes were scanned with an imaging system (Bio-Rad, Hercules, CA, USA).

\section{RNA extraction and qRT-PCR}

TRizol (Takara, Shiga, Japan) was used to extract the intracellular RNA and reverse transcription into cDNA was performed using a reverse transcription kit. The PCR reaction system is $20 \mu \mathrm{L}$, with $\beta$-actin as an internal reference gene, HO-1 (Forward: 5'-3': GCCCACGCATATACCCGCTAC; Reverse: 5'-3': TCTGTCACCCTGTGCTTGACC) and $\beta$-Actin (Forward: 5' -3': CGCGAGTACAACCTTCTTGC; Reverse 5'-3': ATACCCACCATCACACCCTG), qRT-PCR was performed according to the manufacturer's instructions of the commercial kit, and the relative expression level was calculated based on the $\triangle \triangle C T$ method.

\section{Gene chip}

After extracting the total RNA from the liver samples of each group, it was quantified using a NanoDrop ND-2000 (Thermo Scientific) and the RNA integrity was detected with a Agilent Bioanalyzer 2100 (Agilent Technologies, CA, US). After passing the RNA quality inspection, the total RNA was reverse transcribed into double-stranded cDNA and then further synthesized with Cyanine-3-CTP (Cy3)-labeled cRNA. The labeled cRNA was hybridized with the chip, and the original image was obtained by scanning with an 
Agilent Scanner G2505C (Agilent Technologies) after elution. Gene chip detection was performed by OE Biotechnology Co., Ltd., (Shanghai, China). Feature Extraction software (version 10.7.1.1, Agilent Technologies) was used to process the original images to extract the raw data, and GeneSpring GX software (version 14.9, Agilent Technologies) was used to quantile the raw data. The standardized data was filtered, each group of samples was used for comparison, and at least $75 \%$ of the samples marked as detected probes were saved for subsequent analysis. The $P$-value and fold-change value of the t-test were used to screen for differential genes. The screening criteria consisted of up-regulation or downregulation in the fold-change value $\geq 2.0$ and $P$-value $\leq 0.05$. A gene ontology (GO) analysis and Kyoto Encyclopedia of Genes and Genomes (KEGG) analysis were applied to determine the role of these differentially expressed mRNA. The mRNA microarray data were deposited in the NCBI's Gene Expression Omnibus database (GSE167980).

\section{Lymphocyte acquisition, intracellular staining, and flow cytometry}

Following a perfusion of Hanks' solution (Solarbio) into the hepatic portal vein, rat livers were collected and cut to $1 \mathrm{~mm}$ in size and incubated with collagenase IV (Solarbio) at $37^{\circ} \mathrm{C}$ for $30 \mathrm{~min}$, gently ground and passed through a $100 \mu \mathrm{m}$ filter. Single cell suspensions were prepared to obtain rat liver mononuclear cells (MNCs). The following antibodies were used for extracellular staining to identify lymphocytes: CD3 (Biolegend); NK1.1 (Biolegend); CD44 (Thermo Scientific); glucocorticoid-induced tumor necrosis factor receptor (GITR) (Biolegend); CD69 (Biolegend); CD28 (Biolegend); cytotoxic T lymphocyte-associated protein 4 (CTLA-4) (Biolegend); CD160 (Biorbyt, Cambs, UK); B- and T lymphocyte attenuator (BTLA) (Thermo Scientific). Hepatic MNCs were stimulated in vitro with a cell activation cocktail (Biolegend) for 4 - $6 \mathrm{~h}$, fixed, and membrane breaking were performed according to the manufacturer's instructions (Thermo Scientific). The cells were then intracellularly stained with IFN-y (Biolegend) and granzyme B (GZMB) (Biolegend) antibodies. Flow cytometry was performed using a FACS Canto II (BD Biosciences, CA, USA) and the data were analyzed with FlowJo software (ThreeStar, OR, USA).

\section{MNC and HO-1/BMMSCs co-culture}

The HO-1/BMMSCs were seeded into the upper chamber and the liver MNCs were placed into the lower chamber, and stimulate with a-Galactosylceramide (a-GalCer) $(100 \mathrm{ng} / \mathrm{mL})$ [29]. MNCs and HO1/BMMSCs were co-cultured at a 1:1 ratio for $48 \mathrm{~h}$, Brefeldin $\mathrm{A}$ was used to block cytokine release for the final $6 \mathrm{~h}$, and the expression of IFN-y by MNCs was detected by flow cytometry.

\section{Statistical analysis}

SPSS 13.0 (SPSS GmbH, Munich, Germany), GraphPad 8.0 (GraphPad Software Inc., San Diego, CA, US) were used for the statistical analysis. The data were expressed as the mean $\pm S D$, and multi-group analyses were performed by a one-way analysis of variance (ANOVA) for data assessment. A two-tailed Student's t-test or ANOVA were used for comparisons between groups. For the survival analysis of the rats, Kaplan-Meier survival curves were plotted by counting the survival time and a Log-rank (Mantel-Cox) test. $P<0.05$ was considered to indicate statistical significance. 


\section{Results}

\section{Extraction and identification of HO-1/BMMSCs}

BMMSCs were isolated and cultured to the third generation as previously described [16]. BMMSCs transfected with an $\mathrm{HO}-1$ adenovirus remained unchanged morphologically (Figure S2A), and had the ability to induce osteogenic (Figure S2B) and lipogenic (Figure S2C) differentiation in vitro. Flow cytometric identification of surface biomarkers revealed a positive rate of over $99 \%$ for CD29, CD90, and RT1A, and a negative rate of over $99 \%$ for CD34, CD45, and RT1B (Figure S2D - F). This demonstrated that the molecular biology of the HO-1 adenovirus-transfected BMMSCs was not affected. The immunofluorescence exhibited significantly higher red fluorescence intensity in the HO-1/BMMSCs compared to that of the BMMSCs (Figure S2G and H). qRT-PCR (Figure S2I) and Western blot (Figure S2J) results were also confirmed to exhibit significantly higher HO-1 expression in the HO-1/BMMSCs.

\section{The NMP system preserves the donor livers in vitro and allows the colonization of HO-1/BMMSCs}

The normal liver is bright red (Fig. 1A), while the DCD liver is a purplish red color with rounded edges and slight edema (Fig. 1B). After the liver had been preserved using the SCS method, the liver was congested with edema, enlarged in size, and rounded at the edges (Fig. 1C). A stable NMP system was established (Figure S1A and B) and the liver preserved by the NMP method was uniform in color, without edema and congestion (Fig. 1D). After the LT was completed using the "double cuff method", the liver was evenly congested, indicating that the model was successfully established (Fig. 1E and F). Respectively, $1 \times 10^{7}$ GFP/BMMSCs or HO-1/BMMSCs were perfused, and LT was completed. Our previous studies demonstrated that approximately $50 \%$ of BMMSCs could be colonized in the donor liver during the perfusion process [17]. The vivo imaging system was used to detect the liver grafts the fluorescence intensity of the livers perfused with HO-1/BMMSCs was higher than that of BMMSCs (Fig. 1G and H). The liver grafts were obtained, frozen sections were prepared, and the number of $\mathrm{GFP}^{+}$cells in the frozen sections of the HO-1/BMMSCs-perfused liver grafts were significantly higher than that of the BMMSCs (Fig. 1I-K). This finding confirms that in the inflammatory environment after LT, HO-1/BMMSCs survived in significantly higher numbers in the transplanted livers compared to BMMSCs.

\section{HO-1/BMMSCs significantly reduced ACR and improved the recipient prognosis}

The morphological changes of the liver grafts were observed by HE staining (Fig. 2A). At $7 \mathrm{~d}$ post-LT, the SCS group liver grafts were observed to be severely damaged, a large number of hepatocytes disappeared around the vein, a large number of macrophages and lymphocytes had infiltrated, and focal necrosis of the hepatocytes with eosinophilic homogeneity and hemorrhage were locally observed. The liver grafts in the HMP and FK506 groups were significantly improved compared with the SCS, NMP, and BMP groups, there was no obvious necrosis in the liver, and the liver lobular structure was intact, with a 
moderate amount of lymphocytic infiltration surrounding the bile ducts and portal vein. No rats survived in the SCS group at $14 \mathrm{~d}$ post-LT, whereas the liver lobules were more intact in the HBP group, exhibiting neatly arranged hepatocyte strips and low levels of lymphocyte infiltration. The TUNEL results of the liver tissues showed that the apoptotic cells were significantly increased in the SCS and NMP groups compared with other groups, whereas there were no significant differences between the HMP and FK506 groups and both were reduced compared with the BMP group (Fig. 2B and C). The acute cellular rejection scores were not significantly different between the NMP and SCS groups, but significantly increased compared with all other transplantation groups. Moreover, the rejection scores in the HMP and FK506 groups were significantly lower than those in the BMP group $(P<0.05)$; however, there was no significant difference between the HMP and FK506 groups (Fig. 2D). These results indicate that HO-1/BMMSCs combined with NMP preserved the DCD livers in vitro, and HO-1/BMMSCs retained in the liver grafts after transplantation improved the pathology and inhibited ACR, similar to the level of FK506 application.

The median survival time after transplantation in the Sham, HMP, and FK506 groups was $>60 \mathrm{~d}$. All animals survived long-term and no statistical differences were observed regarding a comparison in the survival rate between the groups (Fig. 2E). In the survival analysis conducted using a Log-rank (MantelCox) test, rats in the HBP and FK506 groups had the longest survival time (median survival time: $60>d$ ) compared with the SCS group (median survival time: $1.5 \mathrm{~d}$ ), NMP group (median survival time: $16.5 \mathrm{~d}$ ), BMP group (median survival time: $21.5 \mathrm{~d}$ ), and SCS group. The survival time of the rats in the SCS group was significantly lower than that of the other groups $(P<0.05)$, and the survival time of rats in the BMP group was longer than that of the NMP group but shorter than that of the HBP and FK506 groups $(P<$ $0.05)$. This finding indicates that HO-1/BMMSCs perfused via portal vein could significantly prolong the survival time of the recipient rats after LT, and the application of HO-1/BMMSCs was more effective than BMMSCs.

ALT, AST, ALP, and GGT reflected the level of liver cell injury, and the levels of ALT, AST, and ALP in the HBP group were significantly lower than those in the SCS, NMP, and BMP groups at $7 \mathrm{~d}$ post LT $(P<0.05)$. The difference with the FK506 group was not statistically significant $(P>0.05)$. At 14 d post-LT, no rats in the SCS group survived, and the levels of ALT, AST, and ALP in the HBP group were significantly lower than those in the SCS, NMP, and BMP groups $(P<0.05)$. The levels of ALB responding to hepatic synthetic function were only observed to be significantly lower in the SCS group compared to that of the Sham and HBP groups at $7 \mathrm{~d}$ post-LT $(P<0.05)$. TBil can respond to the excretory function of the liver and to hepatocyte damage. The level of TBIL in the HBP group was higher than that in the Sham group at $7 \mathrm{~d}$ post-LT but significantly lower than that of all the other groups $(P<0.05)$. The level of TBIL in the SCS group was significantly higher than that in all of the other groups. The level of TBIL in the BMP group was lower than that in the NMP group, but higher than that in the HBP group. These findings indicated that HO-1/BMMSCs significantly improved the condition of the recipients' liver function (Fig. 2F).

\section{Gene chip analysis of the differential gene expression in the liver grafts of each group}


We detected the level of mRNA expression in the liver grafts of each group, and analyzed the differential gene expression using gene chip technology, focusing on the NMP, BMP, and HMP groups (Table S1 - 3). The combination of the GO analysis and KEGG analysis revealed that the interaction between cytokines and cytokine receptors, as well as the level of gene expression of Th1- and Th2-related cytokines were significantly different (Fig. 3A - C and S3A - D). We compared the gene expression of cytokines related to ACR (Fig. 3D) and found that the gene expression of Th1 cytokines (e.g., IFN-y, TNFa, and IL-2) related to ACR was significantly lower in the HMP and BMP groups compared with that of the NMP group. The level of gene expression in the above cytokines was lower in the HMP group compared to that of the BMP group. In combination with the analysis of the interaction of cytokine genes associated with ACR performed in a String database (Fig. 3D), we focused on the role of IFN-Y gene expression in HO1/BMMSCs in attenuating ACR. The effect of IFN-y on NKT cells, NK- and Th1- and Th2-related cytokines was assessed.

\section{HO-1/BMMSCs perfused by NMP reduced the proportion of NKT cells and inhibited IFN-Y expression following LT}

Under the guidance of the gene chip analysis results, we next sought to determine the cellular source of IFN- $y$. IFN- $\gamma$ is primarily secreted by NKT and NK cells. Simultaneously, a large number of NKT cells in the liver of rats and NKT cells could secrete IFN- $\gamma$ in large quantities within a few hours of stimulation. In light of the fact that IFN- $y$ can significantly promote ACR, we used flow cytometry to detect the proportion of NKT cells and IFN- $y$ expression in the different recipient tissues. The results showed that following LT, the proportion of NKT cells in the spleen of the NMP group was increased compared with that of the Sham group $(P<0.05)$; however, no significant difference was observed in the other groups $(P>0.05)$. While the proportion of NKT cells in the blood of the NMP group was significantly higher than that of the other groups $(P<0.05)$, no significant difference was observed between the BMP and the HMP groups $(P<$ 0.05). The proportion of NKT cells in the liver of the NMP group was significantly higher than that of the other groups and the HMP group was significantly lower than that of the BMP group $(P<0.05)$ (Fig. 4A). Intracellular staining was used to detect the changes in IFN-y levels in the NKT cells (Fig. 4B). The level of IFN- $y$ in the NKT cells of the NMP group was significantly higher than that of the BMP and HMP groups, and the HMP group was significantly lower than that of the BMP group $(P<0.05)$. These results demonstrated that the DCD donor liver was preserved by NMP combined with HO-1/BMMSCs. The proportion of NKT cells and level of IFN-y in the recipients was significantly reduced following LT. HO1/BMMSCs in the donor livers exhibit a stronger regulatory effect on the NKT cells than BMMSCs.

\section{H0-1/BMMSCs perfusion by NMP can reduce the proportion of NK and CD8 ${ }^{+} \mathrm{T}$ cells in the liver grafts, inhibit their function, and reduce the level of inflammatory factors in the recipients}

Flow cytometry analysis of the proportion of NK cells in the liver grafts revealed that the proportion of the HMP and BMP groups was significantly lower than that of the NMP group, and the HMP group was significantly lower than that of the BMP group $(P<0.05)$ (Fig. 5A). Intracellular IFN-y staining revealed that the level of IFN- $y$ expression in the NK cells of the liver grafts of the HMP group and BMP group was 
significantly lower than that of the NMP group. The HMP group was significantly lower than that of the BMP group $(P<0.05)$ (Fig. 5B). The HO-1/BMMSCs in the DCD donor liver could reduce the proportion of NK cells in the liver grafts after LT, and the inhibitory effect of HO-1/BMMSCs on NK cells was greater than that of the BMMSCs.

Since IFN-y can activate $\mathrm{CD} 8^{+} \mathrm{T}$ cells, we detected the proportion of $\mathrm{T}$ cells in the liver by flow cytometry. The proportion of $\mathrm{CD}^{+} \mathrm{T}$ cells in the liver grafts of the HMP and BMP groups was significantly lower than that of the NMP group, and the proportion of $C D 8^{+} T$ cells in the HMP group was significantly lower than that in the BMP group $(P<0.05)($ Fig. $5 \mathrm{C})$. The level of granzyme B $(\mathrm{GZMB})$ expression reflected the intensity of the toxic effect of $C D 8^{+} T$ cells, and the level of GZMB expression in $C D 8^{+} T$ cells was significantly lower in the liver grafts of the HMP group and BMP group compared with the NMP group,

and it was significantly lower in the HMP group compared with that of the BMP group $(P<0.05)$ (Fig. 5D).

An ELISA was used to detect the ACR-related cytokines, IFN- - , TNF- $a$, and IL-2, and the results showed that the cytokines in the HMP and BMP groups were significantly lower than those of the NMP group, and the HMP group was significantly lower than that in the BMP group $(P<0.05)$ (Fig. 5E). These results suggest that HO-1/BMMSCs can reduce the level of inflammatory factors related to ACR after LT and the effect of HO-1/BMMSCs is greater than that of BMMSCs.

\section{Excessive activation of NKT cells aggravate ACR}

To clarify the role of NKT in the ACR of LT, the rats in the NMP and HMP group the relative specific stimulator of NKT cells were administered a-GalCer $(50 \mu \mathrm{g} / \mathrm{kg})$ by intraperitoneal injection. Our findings showed severe ACR in the transplanted livers of the NMP group, and the application of a-GalCer had no significant effect on the NMP group (Fig. 6A and $B$ ). The histopathology of the liver in the HMP group demonstrated that ACR was significantly reduced (Fig. 6A), the ACR score was reduced (Fig. 6B), and the degree of liver apoptosis was significantly improved (Fig. 6C). However, when a-GalCer was used to activate NKT cells, the level of acute liver rejection was more severe than that in the HMP group. The liver function (Fig. 6D) analysis showed that the level of liver enzymes and TBil were significantly increased under the administration of a-GalCer.

Further detection of NKT cells in the liver grafts revealed that the use of a-GalCer could affect the inhibitory effect of HO-1/BMMSCs on NKT cells and significantly increase the level of IFN- $y$ expression in the NKT cells (Fig. 7A). In the event that a-GalCer induces NKT cell activation, we tested NK cells and $\mathrm{CD}^{+} \mathrm{T}$ cells closely related to ACR. We found that the level of IFN-y in the NK cells and level of GZMB in $\mathrm{CD}^{+} \mathrm{T}$ cells were significantly increased (Fig. 7B and C). In addition, compared to the HMP group, aGalCer caused a significant increase in ACR-related cytokines (e.g., IFN-ץ, TNFa, and IL-2) (Fig. 7D). Our results indicate that the inhibitory effect of HO-1/BMMSCs on NKT regulates ACR. This also suggests that a-GalCer has no effect on ACR after LT; however, in the case of HO-1/BMMSCs-induced transplantation tolerance, NKT cell activation can cause aggravation of ACR. 


\section{Co-culture experiments observed the effect of HO- $1 /$ BMMSCs on IFN- $\gamma$ expression in NKT cells}

We co-cultured HO-1/BMMSCs and liver MNCs in vitro to verify whether it could regulate the function of NKT cells activated by a-GalCer. Our results showed that the level of IFN-y expression in NKT cells was significantly increased following stimulation with a-GalCer. When co-cultured with BMMSCs or HO1/BMMSCs, the level of IFN-y expression was significantly reduced in NKT cells, and the HO-1/BMMSCs inhibited IFN-y expression in NKT cells more significantly than in BMMSCs (Fig. 8A). We detected ACRrelated cytokines (e.g., IFN- $\gamma$, TNF-a, and IL-2) in the culture supernatant, and found that the co-culture of HO-1/BMMSCs with NKT cells could significantly reduce the levels of these cytokines (Fig. 8B). In vitro studies have shown that HO-1/BMMSCs can inhibit IFN-y expression in the NKT cells and reduce the level of cytokines related to ACR.

\section{H0-1/BMMSCs in the liver grafts affects the co-inhibitory receptor expression of NKT cells}

Our previous study confirmed the significant inhibitory effect of HO-1/BMMSCs on the level of IFN-y expression in NKT cells. We further investigated the mechanism of action of HO-1/BMMSCs on NKT cells, the activation of which is triggered by linking semi-invariant TCRs, as well as a series of costimulatory and co-inhibitory receptors. We detected the co-receptor expression on NKT cells in the liver grafts of each group (Fig. 9A and B), and found that NKT cells constitutively expressed a variety of co-stimulatory receptors; however, few differences were observed between the groups. The examination of the coinhibitory receptors revealed that the expression of NKT cell surface expression of the co-inhibitory receptors, BTLA and CD160, differed significantly in each group. In the HMP and BMP groups, BTLA and CD160 expression (proportion of positive cells and MFI) in the NKT cells were significantly higher than that of the NMP group (Fig. 9C and D) and the level of expression in the HMP group was higher than that of BMP group. This finding confirms that the HO-1/BMMSCs in the liver grafts increasing the expression of co-inhibitory receptors BTLA and CD160 on NKT cells, through which inhibitory signals are transmitted to NKT cells, reducing the level of IFN-y secretion from NKT cells and reducing ACR after LT. Moreover, the HO-1/BMMSCs that colonized the transplanted livers had a greater regulatory effect on the NKT cells than the BMMSCs.

\section{The regulation of NKT cell function by HO-1/BMMSCs is achieved through BTLA and CD160}

To further confirm the role of BTLA and CD160 in the regulation of NKT cell function by HO-1/BMMSCs, we extracted MNCs from the normal rat livers using a-GalCer to activate the NKT cells. A blocking antibody was used to block BTLA or/and CD160 in NKT cells, and co-cultured with HO-1/BMMSCs. Our research showed that when BTLA or CD160 was blocked, the level of IFN-ץ expression in the NKT cells increased to varying degrees compared with that of the control group (Fig. 10A). The levels of IFN- $\gamma$, TNF- 
$\mathrm{a}$, and IL-2 in the culture supernatant were also significantly increased compared to that of the control group (Fig. 10B). The level of IFN-y expression in NKT cells was further increased when BTLA and CD160 were both blocked (Fig. 10A), the concentration of IFN-y, TNF-a, and IL-2 were also further increased (Fig. 10B). These results suggest that HO-1/BMMSCs affects the surface expression of BTLA and CD160 on NKT cells, transmitting inhibitory signals to NKT cells and regulating the levels of IFN-y, inhibiting the ACR of LT. Thus, BTLA and CD160 may be co-inhibitory receptors with non-overlapping functions.

\section{Discussion}

ACR following LT remains the primary complication experienced by patients, which directly affects the long-term prognosis. The long-term immunosuppressant regimen is associated with multiple complications, and some patients continue to suffer from ACR under treatment with immunosuppressive agents [9]. In this context, it is necessary to seek new immunomodulatory methods. The interaction between BMMSCs and immune cells can establish a stable and balanced microenvironment through the regulation of innate or acquired immune cells [30]. The intercellular interaction and paracrine response between BMMSCs and immune cells provide a theoretical basis for the treatment of immune-related diseases [31-33]. Numerous studies have shown that BMMSCs play an active role in attenuating the ACR of organ transplantation [34,35]. Currently, studies describing the interaction of BMMSCs with NKT cells in LT remain unclear. BMMSCs are less effective following systemic application and their shorter survival time in target organs continues to challenge the progress of cell therapy.

In this study, NMP was used to preserve the DCD donor liver in vitro while perfusing HO-1/BMMSCs into the portal vein of the donor liver, after which LT was performed. The follow-up results of the BMMSCs remaining in the liver grafts following LT showed that HO-1/BMMSCs can be colonized in large numbers in the liver grafts, and the survival time is longer than that of BMMSCs. Our method solves the problem associated with BMMSCs colonization and the short survival time in target organs, and also avoids complications (e.g., pulmonary embolism and thrombosis), which may be caused by an intravenous application of BMMSCs. Moreover, liver grafts carrying HO-1/BMMSCs exhibited significantly lower ACR after LT, as demonstrated by liver function and histology.

To explore the impact of HO-1/BMMSCs on ACR, we used gene chip technology for analysis and found that the interaction between cytokines and cytokine receptors, as well as Th1 and Th2 cell-related factor gene expression were significantly different. We performed an association analysis of these genes and confirmed that the level of IFN- $y$ expression played a role in the reduction of ACR by HO-1/BMMSCs. In the process of regulating autoimmune responses, NKT cells are essential for microbial defense and initiating an adaptive immune response. There are a large number of NKT cells in the liver sinusoids, and these numbers increase following transplantation [34,35]. NKT cell activation releases a large amount of IFN- $\gamma$, which helps to activate NK cells, $C D 8^{+} T$ cells, and antigen-presenting cells [36, 37]. In addition, IFN$Y$ has been shown to play an important role in allograft rejection, indicating that NKT cells may play a critical role in the ACR of LT. 
Our research shows that HO-1/BMMSCs carried in the liver grafts reduces the number of NKT cells after LT and inhibits IFN-y expression, thereby inhibiting NK and $C D 8^{+} T$ cells activation, and significantly reducing ACR. It is important to note that NKT cells are required for transplantation immune tolerance in some organs or tissues. One heart transplantation study showed that immunosuppressive regimens were effective in the presence of NKT cells and ineffective after the clearance of NKT cells; however, a-GalCer had little effect on rejection in the absence of immunosuppressive regimens [38]. Despite these findings, there have been no reports on the effect of a-GalCer on ACR in LT. Since activated NKT cells release both pro-inflammatory and anti-inflammatory cytokines, they have many different functions in the immune response, and thus their role in the ACR of LT remains unclear.

In our study, the application of a-GalCer did not significantly alleviate ACR in the rats of the NMP group; however, HO-1/BMMSCs significantly alleviated the ACR of LT. The application of a-GalCer could lead to excessive activation of NKT cells, increased IFN-y expression, and aggravated ACR (Fig. 6A). This indicates that the excessive activation of NKT cells promotes the occurrence of ACR of LT, whereas the use of HO-1/BMMSCs inhibits the NKT cell activation and decreases IFN- $y$ expression to reduce the ACR of LT. Thus, the application of a-GalCer has no beneficial for reducing ACR, and may even be harmful.

Activation of NKT cells is triggered by linking semi-invariant TCRs or a series of co-stimulatory and coinhibitory receptors [39]; however, the research on the expression of NKT cell co-receptors in LT is poorly understood. We detected the surface co-receptors of NKT cells in the livers of different treatment groups following LT and found that the levels of CD160 and BTLA expression in the HMP group were significantly increased (Fig. 8). CD160 is considered to be a marker of T cell depletion [40], while BTLA is structurally expressed by NKT cells as an inhibitory receptor that transmits signals from herpes virus entry mediator [41, 42]. CD160 and BTLA share a common ligand that delivers inhibitory signals to NKT cells [43]. Our study revealed that HO-1/BMMSCs reduce IFN- $y$ expression in NKT cells by increasing their surface expression of co-inhibitory receptors, through which inhibitory signals are delivered to NKT cells, revealing a novel target for the regulation of NKT cell function.

Our research shows that NMP is a reliable method for preserving DCD donor livers and provides a new means of applying BMMSCs. Moreover, $\mathrm{HO}-1$ gene transfection enhances the survival of BMMSCs in liver grafts in a complex inflammatory environment following LT. In this respect, liver grafts carrying HO1/BMMSCs significantly attenuated the occurrence of ACR after LT and improved the recipient prognosis. We conducted a preliminary investigation of the role of NKT cells in ACR of LT and confirmed that the regulation of NKT cell surface co-inhibitory receptor expression by HO-1/BMMSCs reduces their level of IFN-y expression. This has a positive effect on the induction of immune tolerance and may provide a novel method of treating ACR of LT.

It also provides a new target of regulation of NKT cells. It should be highlighted that due to the lack of corresponding gene knockout rats, we were unable to verify the mechanism by which ACR of LT induced the complete disappearance of NKT cells. Thus, our animal model may be inadequate to address this issue and further validation in other animal models may be required in future studies.

Page 15/31 


\section{Conclusions}

Our findings demonstrate that NMP can protect the DCD donor liver in vitro. BMMSCs infused via the portal vein using the NMP system can colonize the liver in large quantities. Moreover, HO-1 gene transfection can significantly improve the activity and survival time of BMMSCs in the liver. There was also a significant reduction in ACR following transplantation with donor livers populated with $\mathrm{HO}$ 1/BMMSCs, and an excessive activation of NKT cells was found to play a key role in the ACR. HO1/BMMSCs inhibit the expression of co-inhibitory receptors on the surface of NKT cells through which inhibitory signals are transmitted to reduce the level of IFN-y expression, thereby inhibiting the occurrence of ACR after LT. Together, our results provide a novel method for the application of BMMSCs, as well as a new target for the treatment of ACR and regulation of NKT cells.

\section{Abbreviations}

ACR: acute rejection; a-GalCer: a-Galactosylceramide; ALB: albumin; ALP: alkaline phosphatase; ALT: alanine aminotransferase; AST: aspartate aminotransferase; BMMSCs: bone marrow mesenchymal stem cells; BTLA: B- and T-lymphocyte attenuator;CTLA-4: cytotoxic T-lymphocyte-associated protein 4; DCD: donation after circulatory death; ELISA: enzyme linked immunosorbent assay; GFP: green fluorescent protein; GGT: glutamyl transpeptidase; GITR: glucocorticoid-induced tumor necrosis factor receptor; GO: gene ontology; GZMB: granzyme B; H\&E: hematoxylin \& eosin; HO-1: heme oxygenase-1; IFN: interferon; IL: interleukin; KEGG: Kyoto Encyclopedia of Genes and Genomes; MFI: mean fluorescence intensity; LT: liver transplantation; MNCs: mononuclear cells; NK: natural killer; NMP: normothermic machine perfusion; RAl: rejection activity index; SCS: static cold preservation; TBil: total bilirubin; TCR: T cell receptor; Th: T helper; TNF: tumor necrosis factor; TUNEL: terminal deoxynucleotidyl transferase dUTP nick end labeling

\section{Declarations}

\section{Ethics approval and consent to participate}

All animal protocols were based on the National Institutes of Health "Guide for the Care and Use of Laboratory Animals" (National Institutes of Health publication 85 - 23, Bethesda, MD). Efforts were made to minimize the number of animals used and any discomfort encountered, and all procedures were approved by the Ethics Committee of Tianjin First Central Hospital (license number: 2016-03-A1).

\section{Consent for publication}

Not applicable.

\section{Availability of data and materials}

All datasets generated for this study are included in the manuscript and the supplementary materials. 
The authors declare no competing interests.

\section{Fundings and Acknowledgements}

The work was supported by the National Natural Science Foundation of China (No. 82070639, 81670574, 81441022 and 81270528$)$.

\section{Authors' contributions}

H.S. and Z.S. conceived and designed experiments, and they contributed equally to this work; H.C., L.W, X.T., W.Z.,M.Y., X.T., Y.W., H.S., and Z.S. conducted the experiments and obtained the results. H.C., H.S., and Z.S. sorted and analyzed the results; H.C. and H.S. wrote the draft. H.C. H.S., and Z.S., extensively revised, formatted, and submitted versions of the manuscript. All authors participated in data discussions, and have seen and approved the submitted version of manuscript.

\section{References}

1. Mazzaferro V, Citterio D, Bhoori S, Bongini M, Miceli R, De Carlis L, et al. Liver transplantation in hepatocellular carcinoma after tumour downstaging $(X X L)$ : a randomised, controlled, phase $2 b / 3$ trial. Lancet Oncol. 2020;21:947-56.

2. van Reeven M, Gilbo N, Monbaliu D, van Leeuwen OB, Porte RJ, Ysebaert D, et al. Evaluation of liver graft donation after euthanasia. Jama Surg. 2020.

3. Croome KP, Mathur AK, Mao S, Aqel B, Piatt J, Senada P, et al. Perioperative and long-term outcomes of utilizing donation after circulatory death liver grafts with macrosteatosis: A multicenter analysis. Am J Transplant. 2020;20:2449-56.

4. MacConmara M, Hanish SI, Hwang CS, De Gregorio L, Desai DM, Feizpour CA, et al. Making Every Liver Count: Increased transplant yield of donor livers through normothermic machine perfusion. Ann Surg. 2020.

5. Muller X, Mohkam K, Mueller M, Schlegel A, Dondero F, Sepulveda A, et al. Hypothermic oxygenated perfusion versus normothermic regional perfusion in liver transplantation from controlled donation after circulatory death: first international comparative study. Ann Surg. 2020;272:751-8.

6. Laing RW, Scalera I, Isaac J, Mergental H, Mirza DF, Hodson J, et al. Liver transplantation using grafts from donors after circulatory death: a propensity score-matched study from a single center. Am J Transplant. 2016;16:1795-804.

7. Shaked A, Ghobrial RM, Merion RM, Shearon TH, Emond JC, Fair JH, et al. Incidence and severity of acute cellular rejection in recipients undergoing adult living donor or deceased donor liver transplantation. Am J Transplant. 2009;9:301-8.

8. Levitsky J, Asrani SK, Schiano T, Moss A, Chavin K, Miller C, et al. Discovery and validation of a novel blood-based molecular biomarker of rejection following liver transplantation. Am J Transplant. 2020;20:2173-83. 
9. Adam R, Cailliez V, Majno P, Karam V, McMaster P, Caine RY, et al. Normalised intrinsic mortality risk in liver transplantation: European Liver Transplant Registry study. Lancet. 2000;356:621-7.

10. Nikeghbalian S, Malekhosseini SA, Kazemi K, Arasteh P, Eghlimi H, Shamsaeefar A, et al. The largest single center report on pediatric liver transplantation: experiences and lessons learned. Ann Surg. 2021;273:e70-2.

11. Fatourou EM, Tsochatzis EA. Management of metabolic syndrome and cardiovascular risk after liver transplantation. Lancet Gastroenterol Hepatol. 2019;4:731-41.

12. Shi D, Zhang J, Zhou Q, Xin J, Jiang J, Jiang L, et al. Quantitative evaluation of human bone mesenchymal stem cells rescuing fulminant hepatic failure in pigs. Gut. 2017;66:955-64.

13. Korkmaz-Icoz S, Zhou P, Guo Y, Loganathan S, Brlecic P, Radovits T, et al. Mesenchymal stem cellderived conditioned medium protects vascular grafts of brain-dead rats against in vitro ischemia/reperfusion injury. STEM CELL RES THER. 2021 2021-02-24;12(1):144.

14. Lee RH, Pulin AA, Seo MJ, Kota DJ, Ylostalo J, Larson BL, et al. Intravenous hMSCs improve myocardial infarction in mice because cells embolized in lung are activated to secrete the antiinflammatory protein TSG-6. Cell Stem Cell. 2009;5:54-63.

15. Kurtz A. Mesenchymal stem cell delivery routes and fate. Int J Stem Cells. 2008;1:1-7.

16. Sun D, Cao H, Yang L, Lin L, Hou B, Zheng W, et al. MiR-200b in heme oxygenase-1-modified bone marrow mesenchymal stem cell-derived exosomes alleviates inflammatory injury of intestinal epithelial cells by targeting high mobility group box 3. Cell Death Dis. 2020;11:480.

17. Cao H, Yang L, Hou B, Sun D, Lin L, Song HL, et al. Heme oxygenase-1-modified bone marrow mesenchymal stem cells combined with normothermic machine perfusion to protect donation after circulatory death liver grafts. Stem Cell Res Ther. 2020;11:218.

18. Yang L, Cao H, Sun D, Hou B, Lin L, Shen ZY, et al. Bone marrow mesenchymal stem cells combine with normothermic machine perfusion to improve rat donor liver quality-the important role of hepatic microcirculation in donation after circulatory death. Cell Tissue Res. 2020;381:239-54.

19. Huang H, Lu Y, Zhou T, Gu G, Xia Q. Innate immune cells in immune tolerance after liver transplantation. Front Immunol. 2018;9:2401.

20. Obara H, Nagasaki K, Hsieh CL, Ogura Y, Esquivel CO, Martinez OM, et al. IFN-gamma, produced by NK cells that infiltrate liver allografts early after transplantation, links the innate and adaptive immune responses. Am J Transplant. 2005;5:2094-103.

21. Lan $P$, Fan $Y$, Zhao $Y$, Lou $X$, Monsour HP, Zhang $X$, et al. TNF superfamily receptor OX40 triggers invariant NKT cell pyroptosis and liver injury. J Clin Invest. 2017;127:2222-34.

22. Pellicci DG, Koay HF, Berzins SP. Thymic development of unconventional T cells: how NKT cells, MAIT cells and gammadelta T cells emerge. Nat Rev Immunol. 2020;20:756-70.

23. Brennan PJ, Brigl M, Brenner MB. Invariant natural killer T cells: an innate activation scheme linked to diverse effector functions. Nat Rev Immunol. 2013;13:101-17.

24. Murphy KM, Reiner SL. The lineage decisions of helper T cells. Nat Rev Immunol. 2002;2:933-44. 
25. Godfrey DI, Kronenberg M. Going both ways: immune regulation via CD1d-dependent NKT cells. J Clin Invest. 2004;114:1379-88.

26. Levitsky J, Burrell BE, Kanaparthi S, Turka LA, Kurian S, Sanchez-Fueyo A, et al. Immunosuppression withdrawal in liver transplant recipients on sirolimus. Hepatology. 2020;72:569-83.

27. Wu J, Zheng Z, Chong Y, Li X, Pu L, Tang Q, et al. Immune responsive release of tacrolimus to overcome organ transplant rejection. Adv Mater. 2018;30:e1805018.

28. Kamada N, Calne RY. Orthotopic liver transplantation in the rat. Technique using cuff for portal vein anastomosis and biliary drainage. Transplantation. 1979;28:47-50.

29. Kumar V. NKT-cell subsets: promoters and protectors in inflammatory liver disease. J Hepatol. 2013;59:618-20.

30. Bernardo ME, Fibbe WE. Mesenchymal stromal cells: sensors and switchers of inflammation. Cell Stem Cell. 2013;13:392-402.

31. Shi Y, Wang Y, Li Q, Liu K, Hou J, Shao C, et al. Immunoregulatory mechanisms of mesenchymal stem and stromal cells in inflammatory diseases. Nat Rev Nephrol. 2018;14:493-507.

32. Lv K, Li Q, Zhang L, Wang Y, Zhong Z, Zhao J, et al. Incorporation of small extracellular vesicles in sodium alginate hydrogel as a novel therapeutic strategy for myocardial infarction. Theranostics. 2019;9:7403-16.

33. Yao G, Qi J, Liang J, Shi B, Chen W, Li W, et al. Mesenchymal stem cell transplantation alleviates experimental Sjogren's syndrome through IFN-beta/IL-27 signaling axis. Theranostics. 2019;9:825365.

34. Erpicum P, Weekers L, Detry O, Bonvoisin C, Delbouille MH, Gregoire C, et al. Infusion of third-party mesenchymal stromal cells after kidney transplantation: a phase I-II, open-label, clinical study. Kidney Int. 2019;95:693-707.

35. Dreyer GJ, Groeneweg KE, Heidt S, Roelen DL, van Pel M, Roelofs H, et al. Human leukocyte antigen selected allogeneic mesenchymal stromal cell therapy in renal transplantation: The Neptune study, a phase I single-center study. Am J Transplant. 2020;20:2905-15.

36. Obara H, Nagasaki K, Hsieh CL, Ogura Y, Esquivel CO, Martinez OM, et al. IFN-gamma, produced by NK cells that infiltrate liver allografts early after transplantation, links the innate and adaptive immune responses. Am J Transplant. 2005;5:2094-103.

37. Kronenberg M, Gapin L. The unconventional lifestyle of NKT cells. Nat Rev Immunol. 2002;2:557-68.

38. Seino KI, Fukao K, Muramoto K, Yanagisawa K, Takada Y, Kakuta S, et al. Requirement for natural killer T (NKT) cells in the induction of allograft tolerance. Proc Natl Acad Sci U S A. 2001;98:257781.

39. Pellicci DG, Koay HF, Berzins SP. Thymic development of unconventional T cells: how NKT cells, MAIT cells and gammadelta T cells emerge. Nat Rev Immunol. 2020;20:756-70.

40. Zhang JY, Wang XM, Xing X, Xu Z, Zhang C, Song JW, et al. Single-cell landscape of immunological responses in patients with COVID-19. Nat Immunol. 2020;21:1107-18. 
41. Rodriguez-Barbosa JI, Schneider P, Weigert A, Lee KM, Kim TJ, Perez-Simon JA, et al. HVEM, a cosignaling molecular switch, and its interactions with BTLA, CD160 and LIGHT. Cell Mol Immunol. 2019;16:679-82.

42. Steinberg MW, Cheung TC, Ware CF. The signaling networks of the herpesvirus entry mediator (TNFRSF14) in immune regulation. Immunol Rev. 2011;244:169-87.

43. Kim TJ, Park G, Kim J, Lim SA, Kim J, Im K, et al. CD160 serves as a negative regulator of NKT cells in acute hepatic injury. Nat Commun. 2019;10:3258.

\section{Figures}



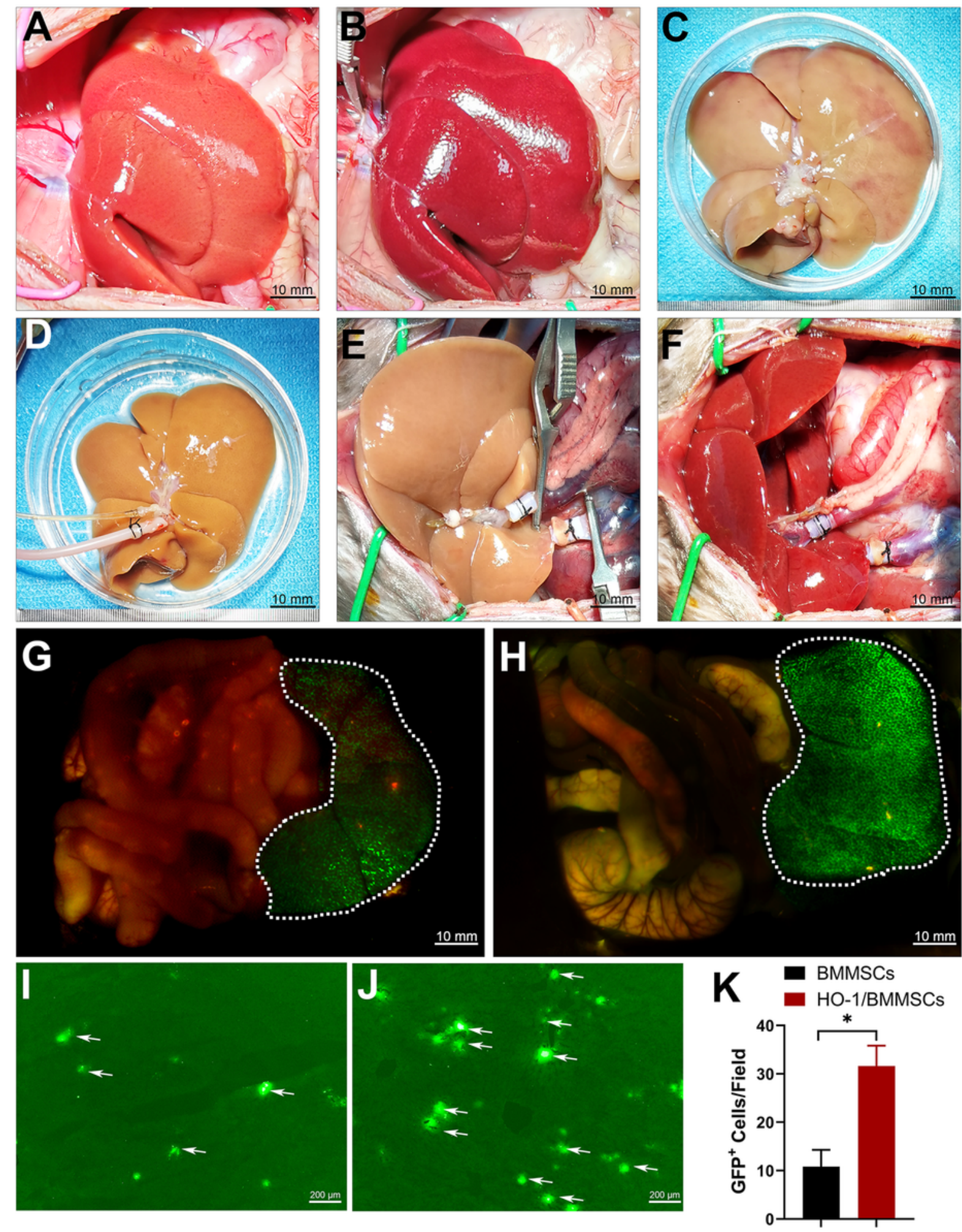

\section{Figure 1}

The DCD LT model and BMMSCs colonization in the liver (A) The general appearance of a normal liver. (B) At 30 minutes after $D C D$, hepatic congestion was severe, exhibiting purple-red appearance, rounded edges, and slight edema. (C) The appearance of the liver preserved by the SCS method was characterized by flaky congestion and edema. (D) The liver preserved by the NMP method has a uniform color without edema and congestion. (E and F) The "two-cuff method" successfully completed rat LT. The in vivo 
imaging system detects the fluorescence intensity of GFP/BMMSCs (G) and HO-1/BMMSCs $(\mathrm{H})$ (indicated by the white dotted line). A fluorescence microscope was used to observe the number of colonizating GFP/BMMSCs (I) and HO-1/BMMSCs $(\mathrm{J})$ in the liver (indicated by the white arrow) on the frozen sections of the liver and the statistical results $(K)$. ${ }^{*} P<0.05$.

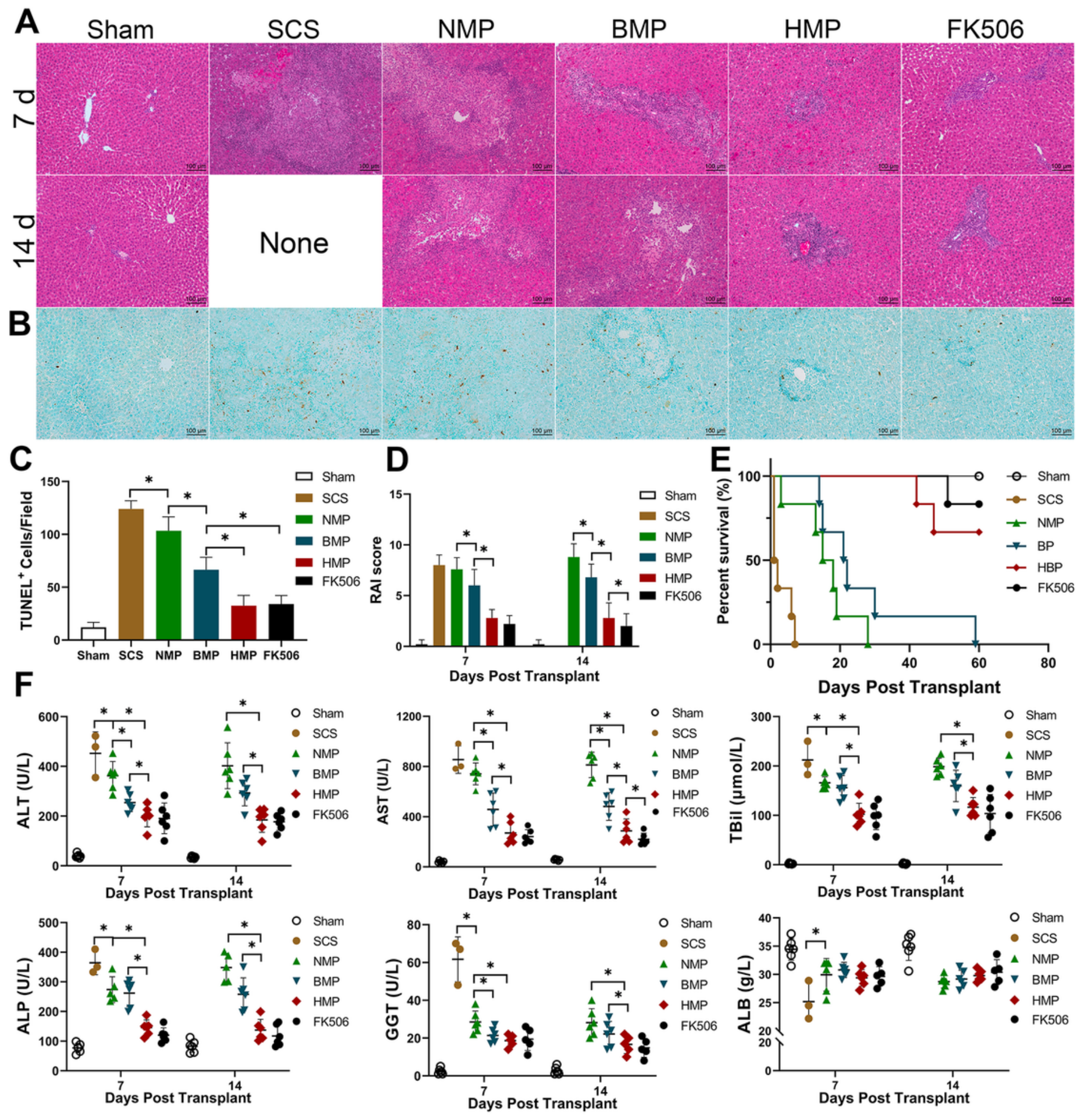

Figure 2 
HO-1/BMMSCs perfused by NMP reduced the ACR after LT (A) Histopathology in the liver of each group after LT. After 7 days of LT, the liver tissue of the NMP group in the SCS group was significantly damaged, in which a large number of liver cells around the vein had disappeared, and a large number of macrophage and lymphocyte infiltration, liver tissue damage in HMP and FK506 groups was mild, with a small amount of lymphocyte infiltration. (B) TUNEL staining of the liver tissue after LT. (C) Statistical results of the number of TUNEL+ cells in each group after LT. The TUNEL+ cells in the HMP group and FK506 group were significantly lower than those in the other operation groups, and there was no significant difference between the HMP group and FK506 group. (D) The RAI score evaluates the degree of ACR after LT. (E) Survival analysis of recipient rats after LT. The median survival time of the Sham, SCS, NMP, BMP, HBP, and FK506 groups were > 60, 1.5, 16.5, 24.5, > 60, and $>60$ d, respectively. (F) Rat liver function after LT. The level of ALT, AST, and TBiL in the HMP and FK506 groups were significantly lower than that in the SCS and NMP groups. There was no significant difference between the HMP and FK506 groups. ${ }^{*} \mathrm{P}<0.05$. 
A
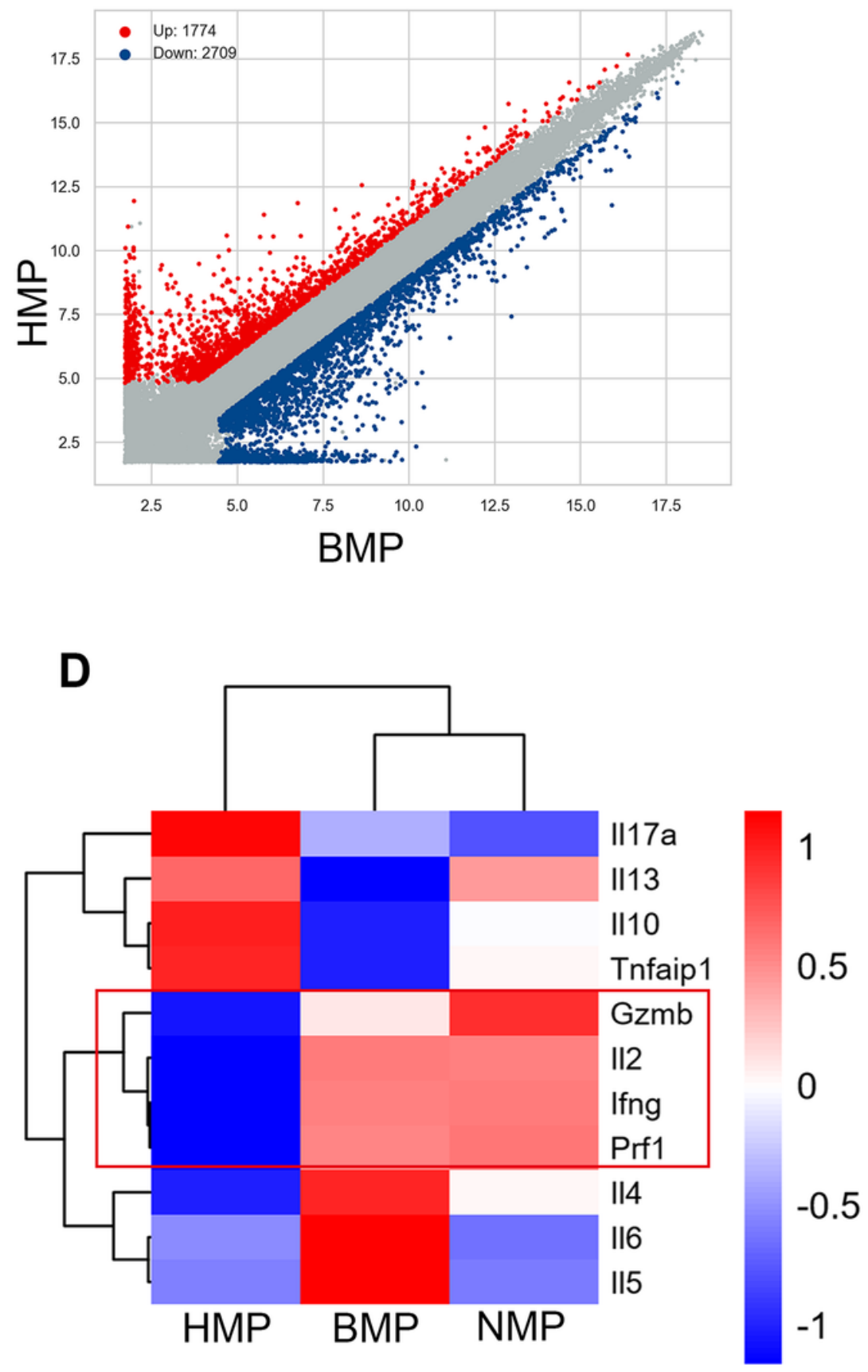
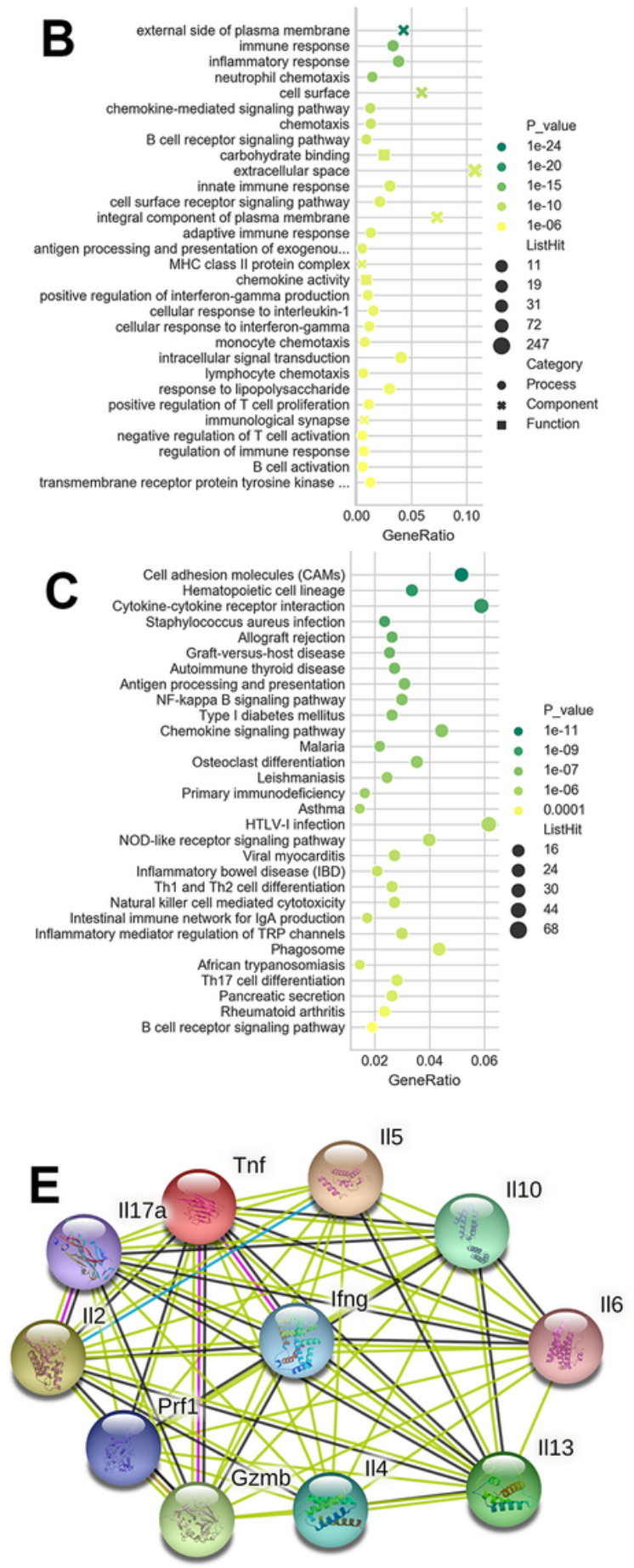

\section{Figure 3}

HO-1/BMMSCs reduced the expression of genes related to ACR of LT (A) The number of differential genes between the HMP group and the BMP group. Go analysis (B) and KEGG (C) analyzes the changes in the signaling pathway in the HMP group and BMP group. Cytokines and cytokine receptor pathways play a key role in ACR after LT. (D) Heat map of cytokine gene expression related to ACR of HNP, BMP, and NMP. (E) Search and analyze the interactions between cytokines related to ACR in the String database. 

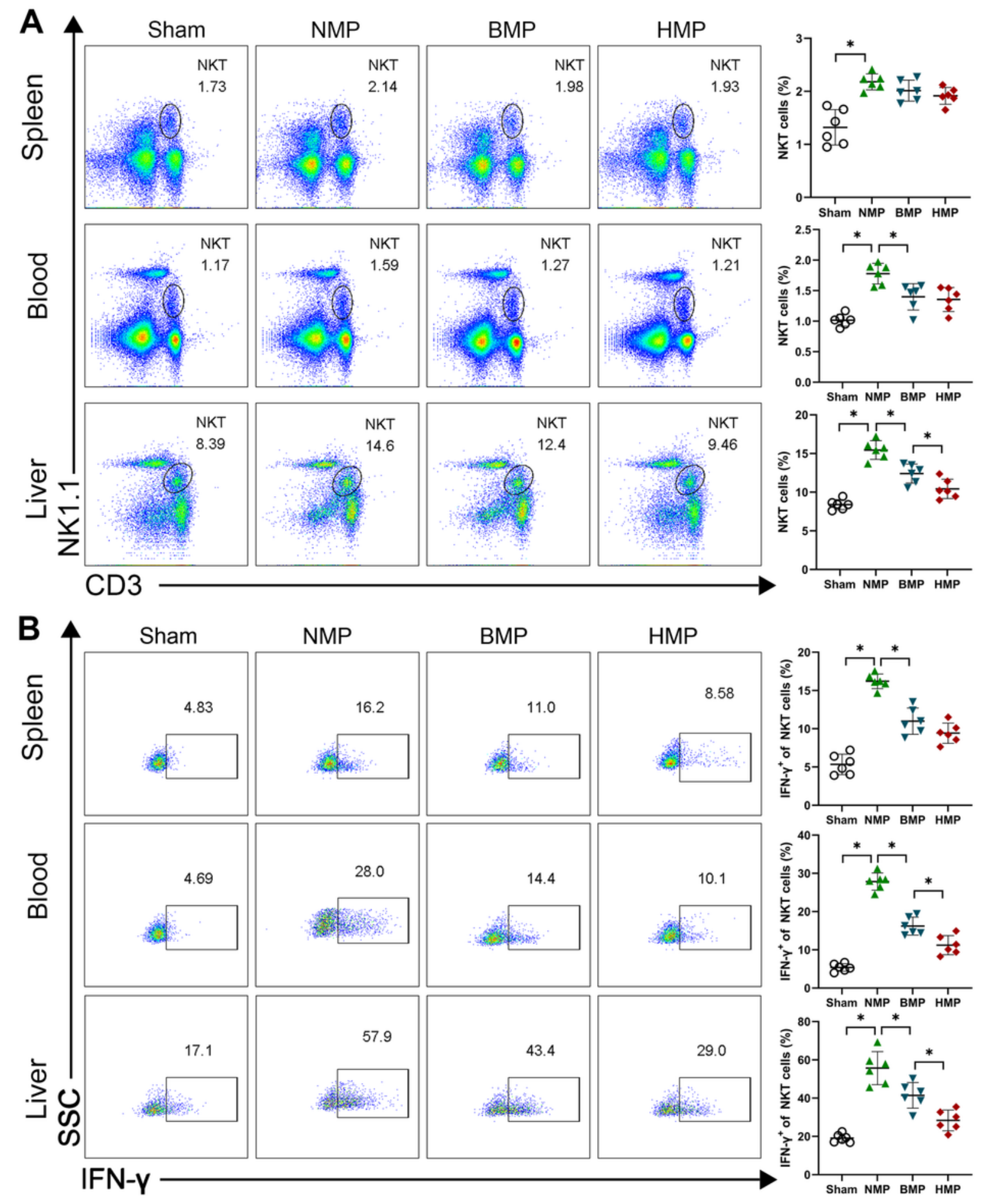

\section{Figure 4}

HO-1/BMMSCs perfused by NMP reduced the proportion of recipient IFN-y expression after LT (A) The proportion of NKT cells in the spleen, blood, and liver. (B) IFN-y expression of NKT cells in the spleen, blood, and liver of recipient rats. IFN-y expression in the NKT cells in the liver of the BMP and HMP groups was significantly lower than that of the NMP group, and the HMP group was lower than the BMP group. $* \mathrm{P}<0.05$. 

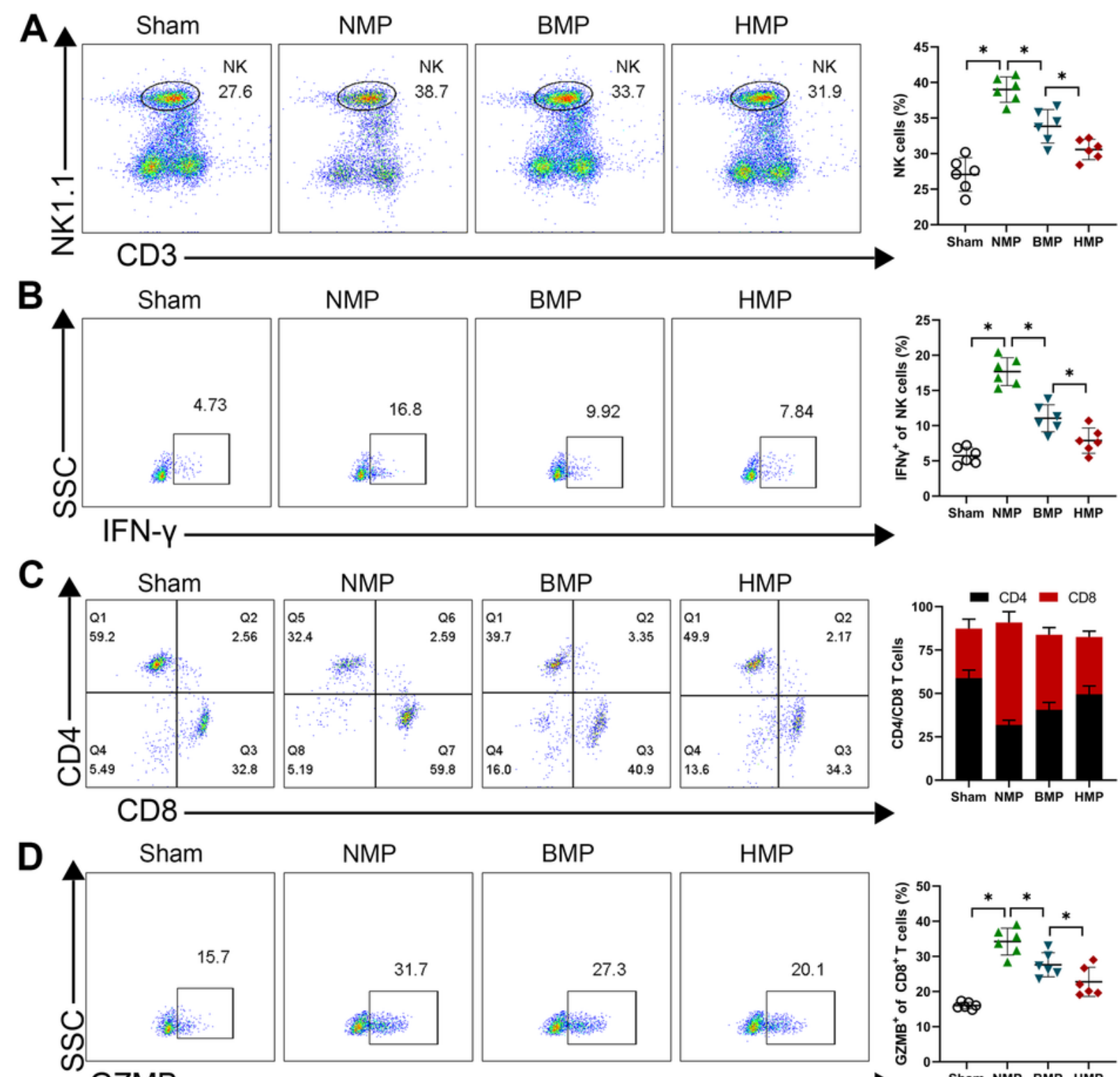

CD8
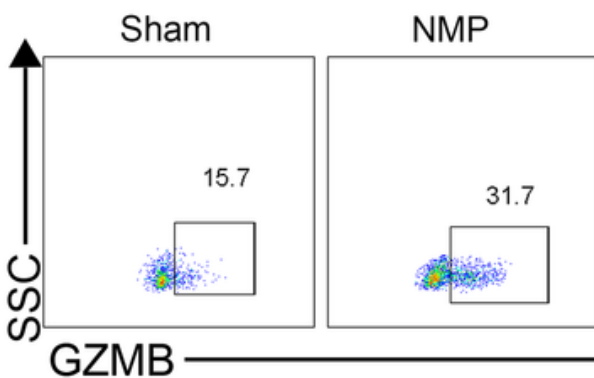

BMP

HMP
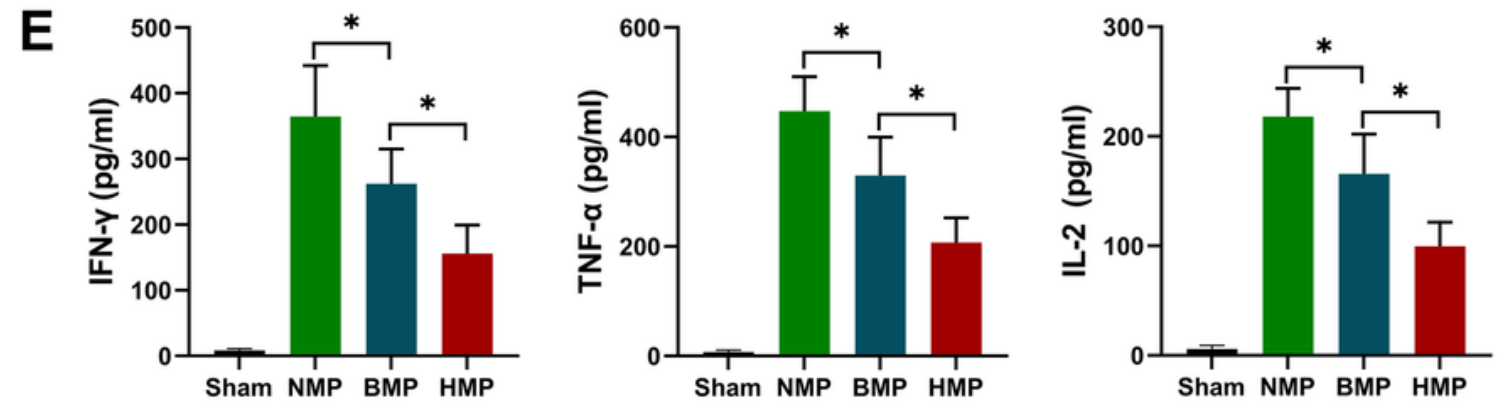

\section{Figure 5}

The proportion and functional changes of NK cells and CD8+ T cells (A) The proportion of NK cells in the liver grafts. The proportion of NK cells in the HMP group was significantly lower than that of the other transplantation groups. (B) The changes in IFN-y expression of NK cells in the liver grafts. (C) The proportion of T cell subsets in the liver grafts. (D) The level of GZMB expression in CD8+ T cells in the liver grafts. (E) Serum levels of ACR cytokines (IFN- $\gamma$, TNF-a, and IL-2). ${ }^{*} P<0.05$. 

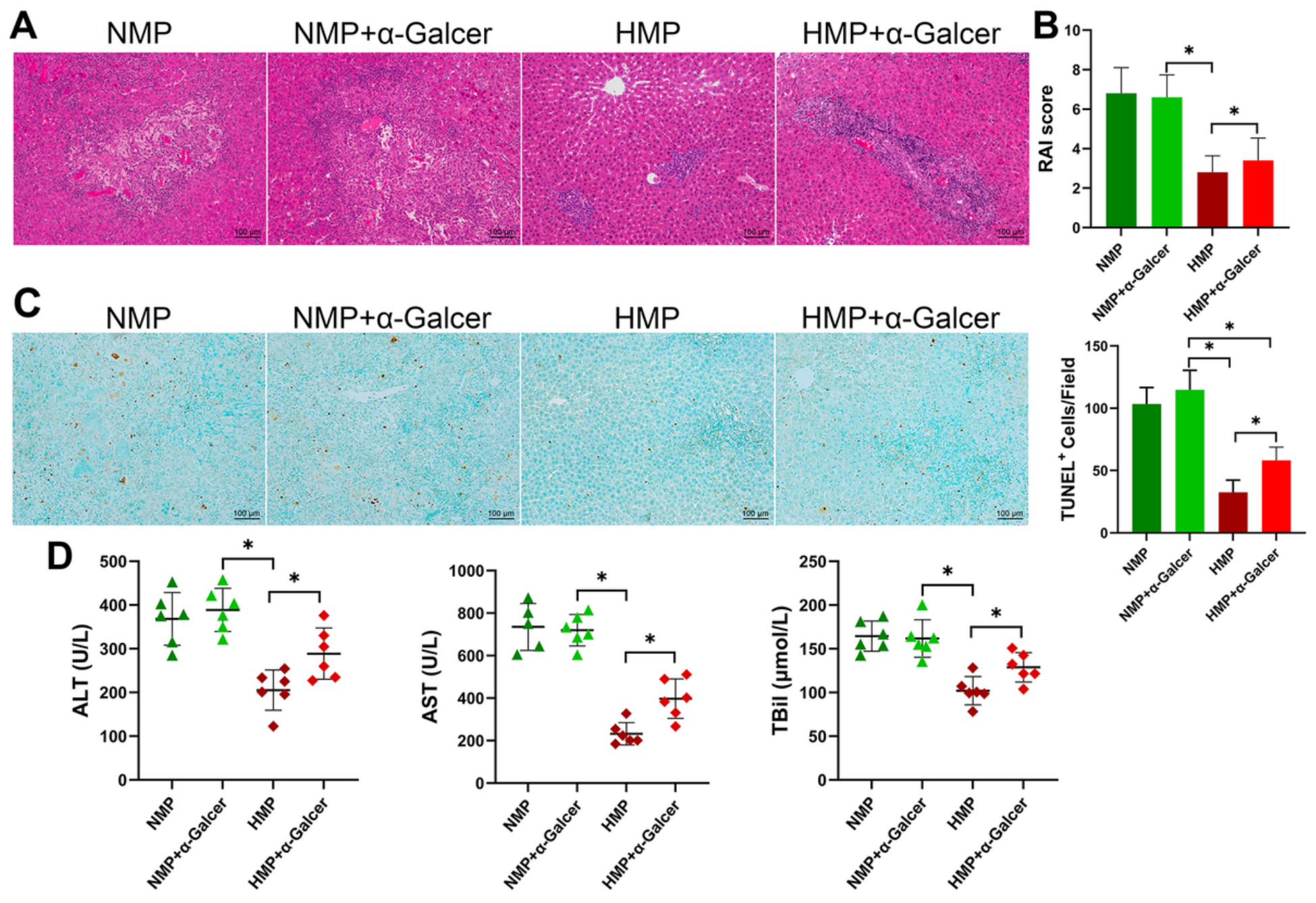

Figure 6

NKT overactivation promotes ACR of LT (A) Rats were injected with a-GalCer after LT. At 7 days postoperation, the rats were sacrificed, and the liver tissues were assessed in the rats. In the case of NKT cell activation, there was an increase in the RAI score (B) and TUNEL+ cells (C). (D) Liver enzyme levels. *P < 0.05 . 


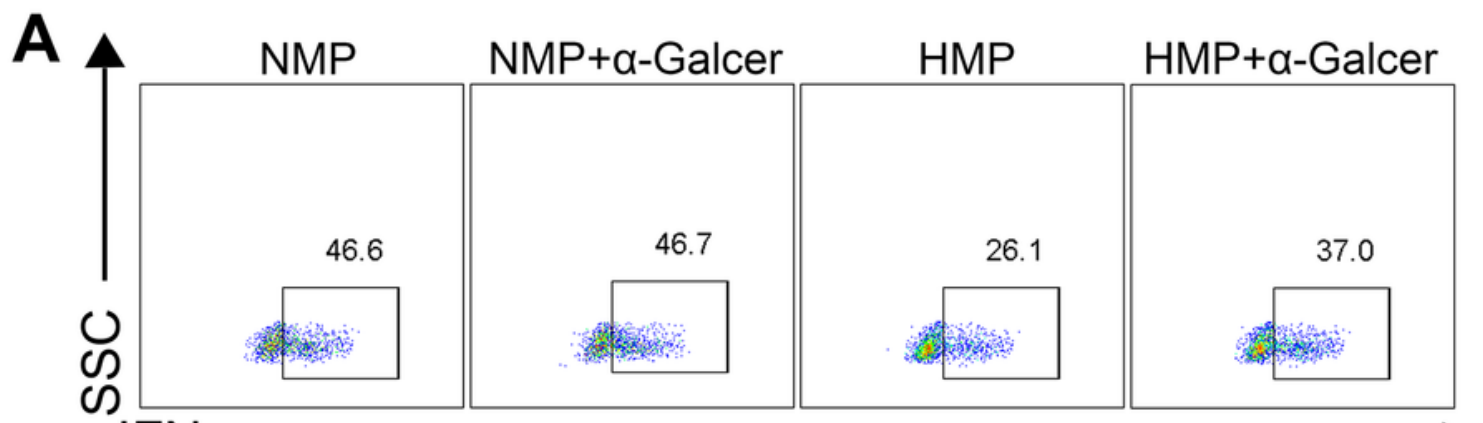

IFN- $\mathrm{Y}$
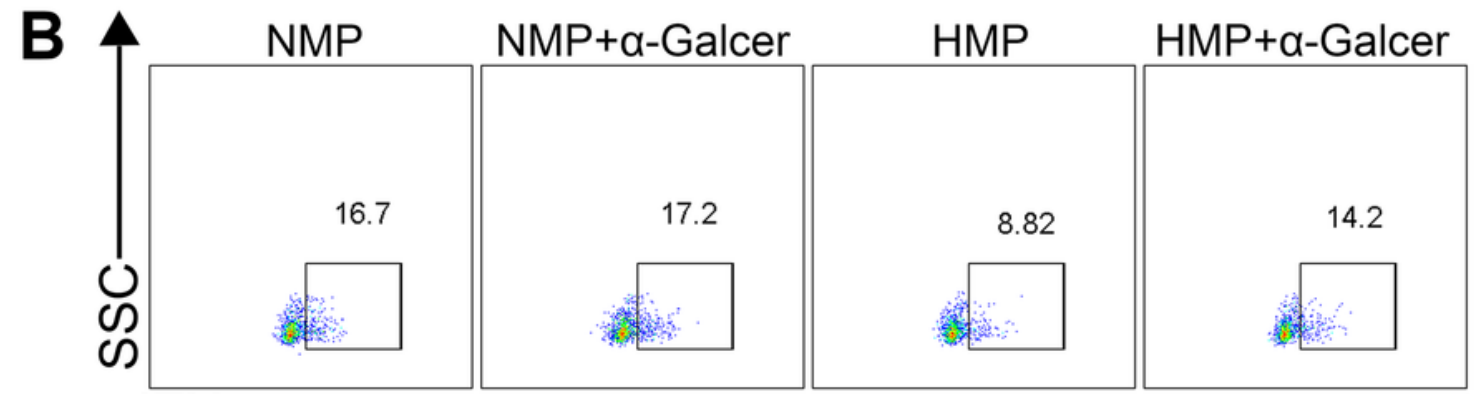

IFN-Y

C

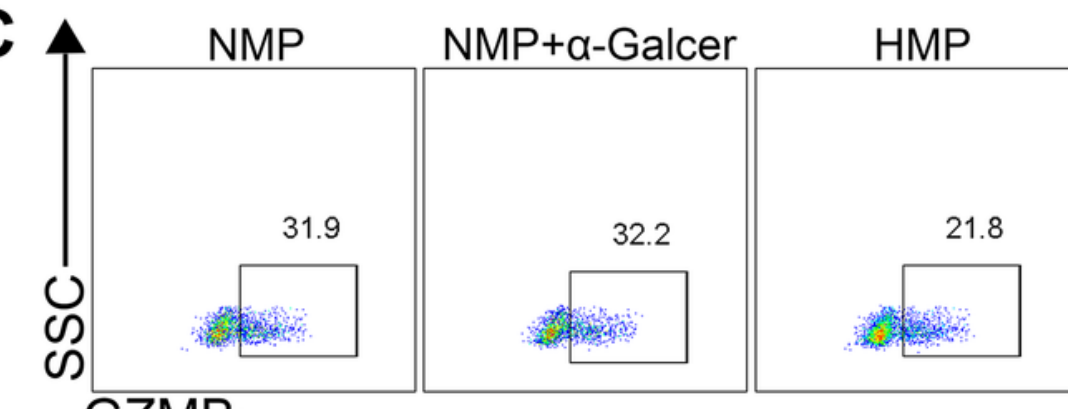

GZMB

D
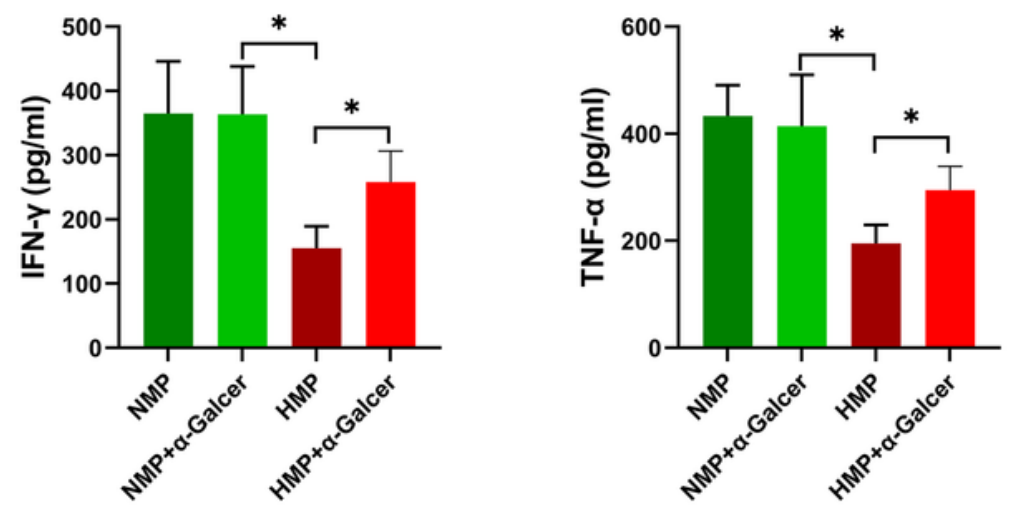

$\mathrm{HMP}+\mathrm{\alpha}-\mathrm{Galcer}$
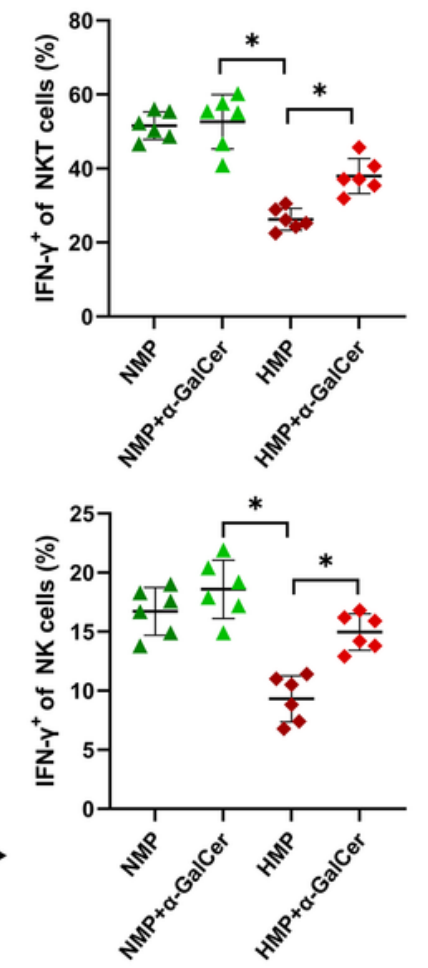

Figure 7

Excessive activation of NKT cells activates NK and CD8+ T cells (A) The level of IFN-y expression in NKT cells. (B) The level of IFN-y expression in NK cells. (C) Level of GZMB expression in CD8+ T cells. (D) Changes in serum levels of ACR-related cytokines. ${ }^{*} P<0.05$. 


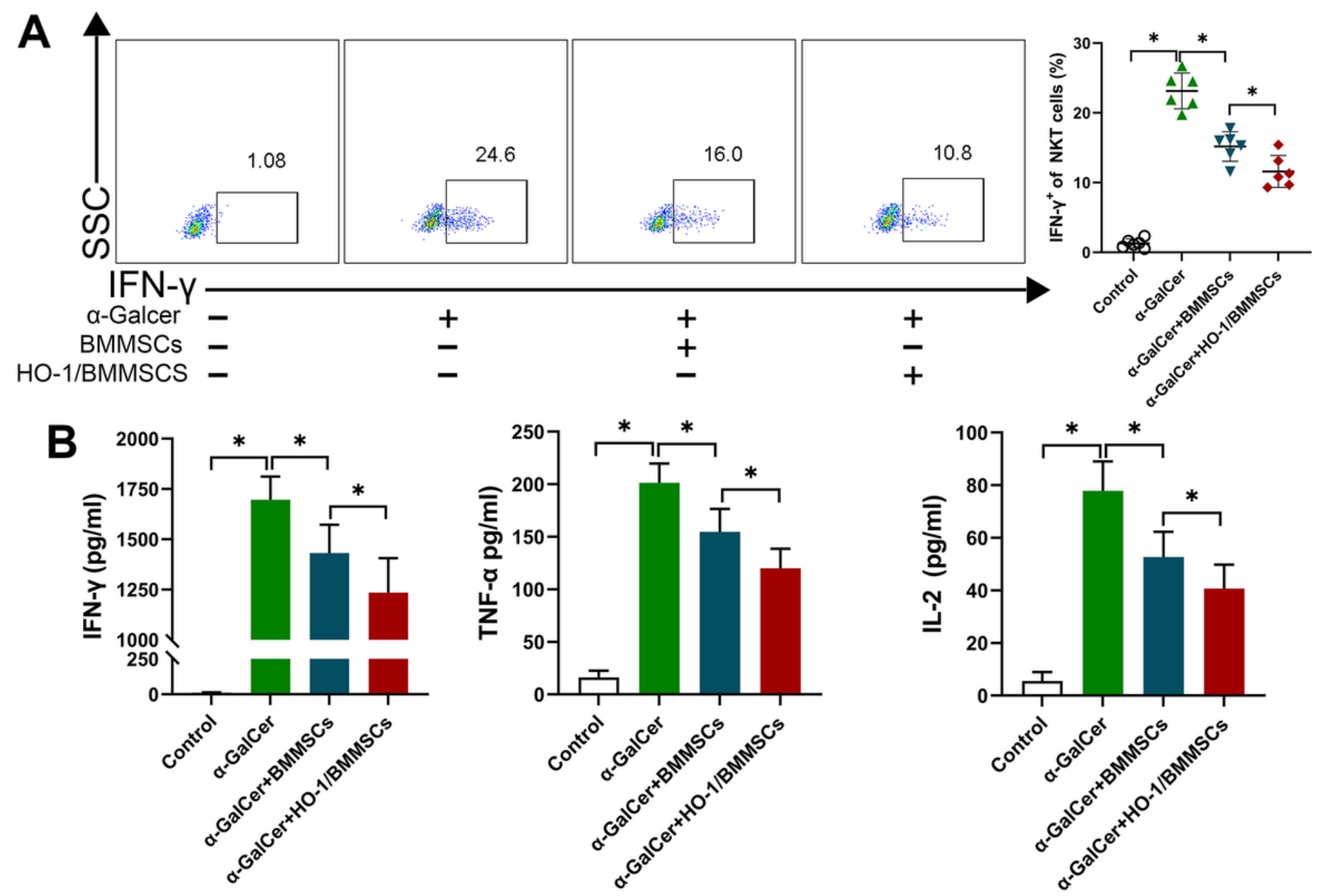

Figure 8

HO-1/BMMSCs inhibited IFN- $\gamma$ expression in NKT cells in vitro (A) The ratio of IFN- $\gamma+$ NKT cells in the liver MNCs of normal rats was analyzed. The ratio IFN- -+ NKT in the HO-1/BMMSCs group was significantly lower than that of the other groups. (B) An ELISA was used to detect the level of IFN- $\gamma$, TNF-a, and IL-2 in the culture supernatant. ${ }^{*} \mathrm{P}<0.05$. 
A Co-stimulatory Receptors
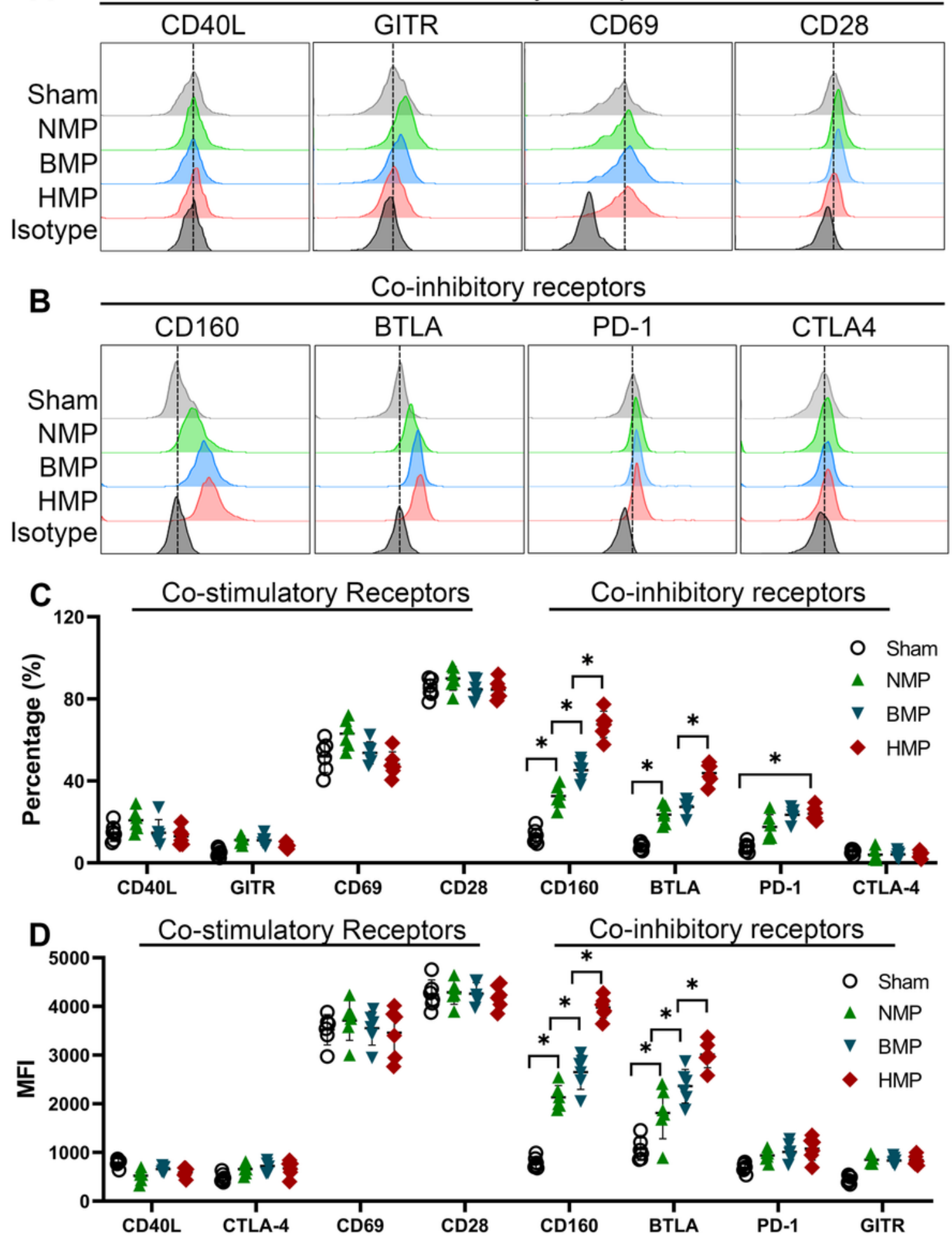

Figure 9

HO-1/BMMSCs affects the level of BTLA and CD160 expression in NKT cells The expression of costimulatory receptors of NKT cells had no significant difference among the groups (A). NKT cell coinhibitory receptors, BTLA and CD160, differed significantly between each group (B). In the HMP and BMP groups, BTLA and CD160 expression in the NKT cells (proportion of positive cells and MFI) (C and D) were significantly higher than that in the NMP group. ${ }^{*} \mathrm{P}<0.05$. 


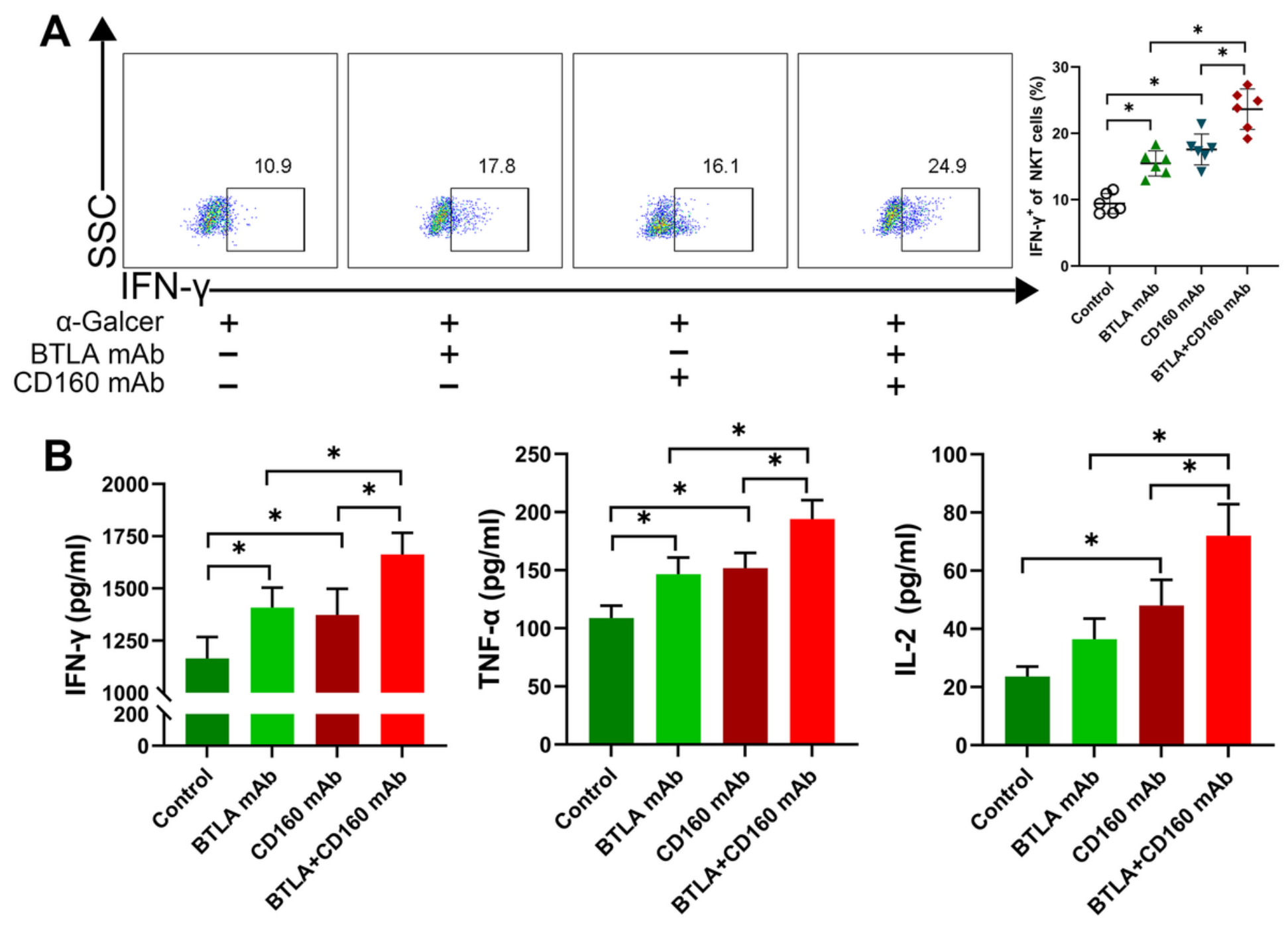

Figure 10

HO-1/BMMSCs had a weakened regulatory effect on NKT cells when the co-inhibitory receptors were blocked. (A) The cells in the control group were incubated with mlgG1 mAb $6 \mathrm{~h}$ in advance, and the other groups were incubated with BTLA or CD160 mAb, and co-cultured with HO-1/BMMSCs for 48 hours. (B) Level of serum cytokines (IFN- $y$, TNF-a, and IL-2). *P $<0.05$.

\section{Supplementary Files}

This is a list of supplementary files associated with this preprint. Click to download.

- SupplementaryFigures.pdf

- SupplementaryTables.docx 